\title{
Investigating the Short-Circuit Problem Using the Planarity Index of Complex q-Rung Orthopair Fuzzy Planar Graphs
}

\author{
Abrar Hussain $\left(\mathbb{D},{ }^{1}\right.$ Ahmed Alsanad $\mathbb{D}^{2},{ }^{2}$ Kifayat Ullah $\mathbb{D}^{1},{ }^{1}$ Zeeshan Ali $\left(\mathbb{D},{ }^{3}\right.$ \\ Muhammad Kamran Jamil $\mathbb{D},{ }^{1}$ and Mogeeb A. A. Mosleh $\mathbb{D}^{4}$ \\ ${ }^{1}$ Department of Mathematics, Riphah Institute of Computing \& Applied Sciences (RICAS), \\ Riphah International University (Lahore Campus), 54000 Lahore, Pakistan \\ ${ }^{2}$ STC's Artificial Intelligence Chair, Department of Information Systems, College of Computer and Information Sciences, \\ King Saud University, Riyadh 11543, Saudi Arabia \\ ${ }^{3}$ Department of Mathematics \& Statistics, International Islamic University Islamabad, Islamabad 44000, Pakistan \\ ${ }^{4}$ Faculty of Engineering and Information Technology, Taiz University, Taiz 6803, Yemen
}

Correspondence should be addressed to Ahmed Alsanad; aasanad@ksu.edu.sa, Kifayat Ullah; kifayat.ullah@riphah.edu.pk, and Mogeeb A. A. Mosleh; mogeebmosleh@taiz.edu.ye

Received 12 April 2021; Accepted 22 June 2021; Published 22 July 2021

Academic Editor: Ahmed Mostafa Khalil

Copyright $\odot 2021$ Abrar Hussain et al. This is an open access article distributed under the Creative Commons Attribution License, which permits unrestricted use, distribution, and reproduction in any medium, provided the original work is properly cited.

Planar graphs play an effective role in many practical applications where the crossing of edges becomes problematic. This paper aims to investigate the complex q-rung orthopair fuzzy (CQROF) planar graphs (CQROFPGs). In a CQROFPG, the nodes and edges are based on complex QROF information that represents the uncertain knowledge in the range of unit circles in terms of complex numbers. The motivation in discussing such a topic is the wide flexibility of QROF information in the expression of uncertain knowledge compared to intuitionistic and Pythagorean fuzzy settings. We discussed the complex QROF graphs (CQROFGs), complex QROF multigraphs (CQROFMGs), and related terms followed by examples. Furthermore, the notion of strength and planarity index (PI) of the CQROFPGs is defined and exemplified followed by a study of strong and weak edges. We further defined the notion of complex QROF face (CQROFF) and complex QROF dual graph (CQROFDG) and exemplified these concepts. A study of isomorphism, coweak and weak isomorphism, is set up, and some results relating to the CQROFPG and isomorphisms are explored using examples. Furthermore, the problem of short circuits that results due to crossing is discussed because of the proposed study where an algorithm based on complex QROF (CQROF) information is presented for reducing the crossing in networks. Some advantages of the projected study over the previous study are observed, and some future study is predicted.

\section{Introduction}

Multiattribute decision making (MADM), pattern recognition, clustering, shortest path problems, and networking problems are some famous areas of real life where uncertainties are involved. In these situations, fuzzy set and its extended frameworks can be very beneficial, and many such problems are discussed in different fuzzy frameworks. In short, where there is uncertainty, there is room for a fuzzy set and its extensions to model such problems. To discuss several real-life problems involving complex information, the conception of the intuitionistic fuzzy set (IFS) based on falsity degree endowed with a truth degree was introduced by Atanassov [1]. In an IFS, the addition of the falsity degree enhanced the conception of the FS (introduced by Zadeh) [2] where only one aspect of the uncertain situation is expressed by a truth degree. This model of IFS is based on a certain restriction where the total of both falsity and truth degree lies in $[0,1]$ that restricts the allocation of the falsity and truth degrees. This barrier of allocation of the degrees of falsity and truth was relaxed by Yager [3] by introducing the conception of the Pythagorean FS (PyFS) where the total of 
the squares of both degrees allows to be in $[0,1]$. The PyFS is a significant improvement in fuzzy settings, but still, allocation of the degrees of falsity and truth needed more flexibility which was granted by the conception of the QROF set (QROFS) by Yager [4]. The layout of the QROFS removes any kind of barrier once and for all whenever information needs to be portrayed by duplets, i.e., falsity and truth degrees. A significant amount of research is performed in the frame of these duplets, and one is referred to [5-10] for some recent accomplishment.

The earlier discussed fuzzy layouts including FS, IFS, PyFS, and QROFS take degrees of falsity and truth from the real unit interval $[0,1]$. This was generalized by Ramot et al. [11] where complex numbers are used to portray uncertain information from a unit circle by the notion of complex FS (CFS). In the settings of CFS, truth degrees of the information are expressed by those complex numbers that lie in a unit circle (complex numbers with magnitude less than or equal to 1). This was a new addition in the fuzzy environment and was followed by many researchers for practical usage. This notion of CFS was followed by Alkouri and Salleh [12], Ullah et al. [13], and Liu et al. [14, 15] who proposed the layouts of the complex IFS (CIFS), complex PyFS (CPyFS), and complex QROFS (CQROFS), respectively. These investigations are quite familiar with the work carried out in $[1,3,4]$ with the rules that the CIFS allows the total of complex truth and falsity degrees in the range $[0,1]$ and the CPyFS allows the total of the squares of the complex truth and falsity degrees in the range $[0,1]$, while the CQROFS allows the total of the $\mathrm{q}^{\text {th }}$ powers of the complex falsity and truth degrees in the range $[0,1]$. In these investigations, if a complex truth or falsity degree is expressed in terms of polar form, say $\left(r . e^{i \theta}\right)$, then $r$ and $\theta$ denote the amplitude and phase terms, respectively. Although these conceptions are very recently introduced, still a great amount of attraction is given to these notions and some remarkable work has been carried out in [16-24].

The theory of fuzzy graph (FG) was discovered by Rosenfeld [25] where the nodes and edges are defined in terms of the membership degrees. There were some cases where the falsity degree needs to be employed along with truth degree and the theory of FG was not able to cope with such issues; for this, the theory of intuitionistic fuzzy graph (IFG) was discovered by Parvathi and Karunambigai [26] by associating the falsity degree with FG. The theory of IFGs is an advanced and elastic version than FGs to manage awkward and complicated information in realistic issues. A deep study about the investigations of the IFGs is carried out in [27]. Moreover, Akram et al. [28] modified the theory of IFG by discussing the theory of Pythagorean FG (PyFG) where the restraint imposed on the IFG was improved by allowing the total of the squares of the truth and falsity degrees in the range $[0,1]$. Furthermore, Habib et al. [29] modified the theory of PyFG to discover the theory of QROF graphs (QROFGs) by improving the restriction on duplets of information by allowing the sum of the q-powers of the truth and falsity grades in the range $[0,1]$. The theory of QROFG was further studied and investigated by [30] where the notion of hypergraphs is introduced in the frame of QROFGs.

Motivated by the work of Ramot et al. [11] and Rosenfeld [25], the notion of complex FG (CFG) was introduced by Thirunavukarasu et al. [31]. In CFGs, the degrees of truth are described using complex numbers instead of real values from $[0,1]$. This notion of CFG cannot be applicable in some cases; for this, the theory of complex IFG (CIFG) was discovered by Yaqoob et al. [32] by discussing the complexvalued falsity degree in the environment of CFG. The theory of CIFG is an enhanced version of the CFG to manage awkward and complicated information in realistic issues. Moreover, Akram and Naz [33] modified the theory of CIFG to discover the theory of complex PyFG (CPyFG) by improving the condition of CIFG, i.e., the sum of the squares of the complex-valued truth and complex-valued falsity grades ranges in $[0,1]$. The theory of CPyFG is more flexible than CIFG to manage awkward and complicated information in realistic issues. A comprehensive study is established on the frame of CPyFG in [34, 35].

$\mathrm{PI}$ is one of the widely discussed characteristics in graph theory which has some potential application in electric circuit theory. A planar graph is a graph that has no crossings of its edges, and this concept is very beneficial in reducing short circuits in electric networks. In the framework of FGs, IFGs, and PyFGs, the PI is discussed comprehensively with its applications, and for details, one can see [36-40]. The concept of planarity is also investigated in several other frameworks including CPyFGs in [29, 41, 42]. There are some issues that cannot be resolved by using the $\mathrm{CPyFG}$, for instance, if a decision maker provides information that cannot be handled by the CPyFG due to its constraint that the sum of the squares of both grades cannot exceed from unit interval. For managing such types of issues, we aim to enhance the theory on the PI of the FG, IFG, PyFS, CIFG, and CPyFG by investigating the theory of the PI of the complex QROFG (CQROFG). The theory of PI in the environment of the CQROFG has two main advantages over previous work as it discusses two aspects of opinion by falsity and truth degree. Secondly, it represents uncertain information with the help of complex truth and falsity degrees with greater flexibility. The summary of the proposed work is discussed as follows:

(1) CQROFPG is an enhanced version of the QROFPG to cope with awkward and complicated information in realistic decision issues. In this investigation work, the idea of CQROFPG and its related ideas are discovered and verified with the help of examples.

(2) The planarity of these outlines relies on the fact that the information is described in terms of a complex number instead of crisp values from $[0,1]$. Here, the contemplations of CQROFMGs, CQROFPGs, and some perceived pieces of these graphs are presented by examining the complex QROF planarity using weak and strong edges.

(3) A close-by association is set up among CQROFPGs and dual graphs. Discussion about the nonplanarity 
of graphs and the thoughts of coweak isomorphism, isomorphism moreover, weak isomorphism for CQROFPGs is in like manner added.

(4) By using numerical examples, the applications of the discovered theories are also utilized. To find the reliability and effectiveness of the presented work, we discussed the comparative study, advantages, and geometrical representations of the explored approaches.

The manuscript is organized as follows: In Section 2, we recall the ideas of the QROFS, QROFG, and CQROFSs and some other basic terms. In Section 3, the conception of the complex q-rung orthopair fuzzy relation (CQROFR), CQROFG, complex QROF multiset (CQROFMS), and CQROFMG and the ideas of strong and effective edges are elaborated. Section 4 is based on notions of strong edges in the CQROFGs, CQROFPG, and PI where examples are provided in support of these notions. In Section 5, the concepts related to CQROFF and CQROFDGs are discussed along with related results supported by examples. In Section 6, the isomorphism between two CQROFPGs is established where the coweak and weak isomorphic relations are investigated with the help of examples. In Section 7, we present an application of the PI of CQROFPGs in short-circuit problems with the help of illustrative examples. In Section 8, we comparatively examine the advantages of the CQROFPGs over the previous study followed by some conclusive remarks in Section 9.

\section{Preliminaries}

The purpose of this section is to recall some basic terms and notions based on which we proposed the new concepts and results. We discussed some basic definitions of the PyFS [2], CPyFS [13], CPyFG [36], CPyFMS [36], QROFS [4], QROFG [30], and CQROFS [14] in this section. Throughout this paper, $\mu, \alpha$ and $\nu, \beta$ denote the truth and falsity degrees of the information respectively, whose values are assigned from $[0,1]$. Furthermore, $\mathscr{X}$ denotes a universal set and $m$ be its arbitrary element.
Definition 1 (see [2]). A PyFS is of the form

$$
T=\left\{\left(m,\left(\mu_{T}(m), \nu_{T}(m)\right)\right): 0 \leq \mu_{T}^{2}(m)+\nu_{T}^{2}(m) \leq 1\right\} .
$$

Definition 2 (see [13]). A CPyFS is of the form

$$
T=\left\{\begin{array}{c}
\left(m,\left(\mu_{T}(m) e^{2 \pi i \alpha_{T}(m)}, \nu_{T}(m) \cdot e^{2 \pi i \beta_{T}(m)}\right)\right): i=\sqrt{-1} \\
0 \leq \mu_{T}^{2}(m)+\nu_{T}^{2}(m) \leq 1,0 \leq \alpha_{T}^{2}(m)+\beta_{T}^{2}(m) \leq 1
\end{array}\right\} .
$$

Definition 3 (see [36]). A CPyFG on $\mathscr{X}$ is a pairG $=(T, S)$, where $T$ is a CPyF vertex set on $\mathscr{X}$ and $S$ is a CPyF relation on $E \subseteq \mathscr{X} \times \mathscr{X}$ such that

$$
\begin{aligned}
& \mu_{S}(x y) \leq \mu_{T}(x) \wedge \mu_{T}(y), \\
& v_{S}(x y) \leq \nu_{T}(x) \vee v_{T}(y), \\
& \alpha_{S}(x y) \leq \alpha_{T}(x) \wedge \alpha_{T}(y), \\
& \beta_{S}(x y) \leq \beta_{T}(x) \vee \beta_{T}(y),
\end{aligned}
$$

where $0 \leq \mu_{S}^{2}(x y)+\nu_{S}^{2}(x y) \leq 1,0 \leq \alpha_{S}^{2}(x y)+\beta_{S}^{2}(x y) \leq 1$, for all $x, y \in \mathscr{X}$ and for all $x y \in E$.

Definition 4 (see [36]). A CPyFMS $T$ is characterized in a way where falsity degree $\mathscr{F} \bigoplus_{T}(m)$ and truth degree $\mathrm{T}_{T}(m)$ functions give every object $m \in \mathscr{X}$ degrees of truth and falsity in $\mathbb{C}$ given by $\mathrm{T}_{T}(m): \mathscr{X} \longrightarrow Q$ and $\mathscr{F} \bigoplus_{T}(m)$ : $X \longrightarrow Q^{\prime}, \quad$ that is, $Q=\{c: c \in \mathbb{C},|c| \leq 1\}, Q^{\prime}=\left\{c^{\prime}\right.$ : $\left.c^{\prime} \in \mathbb{C},\left|c^{\prime}\right| \leq 1\right\} \quad$ and $\quad c=\mu_{T}^{p}(m) e^{2 \pi \alpha_{T}^{p}(m)} \quad$ and $\quad C^{\prime}=v_{T}^{\not p}$ $(m) e^{2 \pi \beta_{T}^{p}(m)}$. The elements of $\mathrm{T}_{T}(m)$ i.e. $\mu_{T}^{1}(m) e^{2 \pi i \alpha_{T}^{1}(m)}$, $\mu_{T}^{2} e^{2 \pi i \alpha_{T}^{2}(m)}, \ldots, \mu_{T}^{\mathfrak{s}}(m) e^{2 \pi \alpha_{T}^{3}(m)}$, where $\mu_{T}^{1}(m) \geq \mu_{T}^{2}(m) \geq$ $\cdots \geq \mu_{T}^{\mathfrak{s}}(m) \quad$ (for amplitude terms) and $\alpha_{T}^{1}(m) \geq \alpha_{T}^{2}$ $(m) \geq \cdots \geq \alpha_{T}^{\mathfrak{s}}(m)$ (for phase terms). A similar pattern can be followed for the elements of $\mathscr{F} \bigoplus_{T}(m)$ such that $0 \leq\left(\mu_{T}^{p}(m)\right)^{2}+\left(\nu_{T}^{p}(m)\right)^{2} \leq 1 \quad$ and $\quad 0 \leq\left(\alpha_{T}^{p}(m)\right)^{2}+$ $\left(\beta_{T}^{p}(m)\right)^{2} \leq 1$ for all $p=1,2, \ldots, \mathfrak{g}$. For convenience, we have

$$
\left\{\left(m \cdot \mu_{T}^{p}(m) e^{2 \pi \alpha_{T}^{p}(m)}, v_{T}^{\not p}(m) e^{2 \pi \mu \beta_{T}^{p}(m)}, p=1,2, \ldots, \mathfrak{g}\right): m \in \mathscr{X}\right\}
$$

Definition 5 (see [4]). A QROFS is of the form

$$
T=\left\{\left(m,\left(\mu_{T}(m), \nu_{T}(m)\right)\right): 0 \leq \mu_{T}^{q}(m)+v_{T}^{q}(m) \leq 1, \quad q \in \mathbb{Z}^{+}\right\} .
$$

Definition 6 (see [30]). A QROFG on a nonempty set $\mathscr{X}$ is a pair $G=(T, S)$ where $T$ is a QROF vertex set on $\mathscr{X}$ and $S$ is a QROF relation on $E \subseteq \mathscr{X} \times \mathscr{X}$ such that

$$
\begin{aligned}
& \mu_{S}(u v) \leq \mu_{T}(u) \wedge \mu_{T}(v), \\
& v_{S}(u v) \leq \nu_{T}(u) \vee \nu_{T}(v),
\end{aligned}
$$


where $\mu_{S}: \mathscr{X} \times \mathscr{X} \longrightarrow[0,1]$ and $\nu_{S}: \mathscr{X} \times \mathscr{X} \longrightarrow[0,1]$ with Definition 7 (see [14]). A CQROFS is of the form $0 \leq \mu_{S}^{q}(u v)+v_{S}^{q}(u v) \leq 1, u, v \in \mathscr{X}, u v \in E, q \in \mathbb{Z}^{+}$.

$$
T=\left\{\begin{array}{c}
\left(m,\left(\mu_{T}(m) e^{2 \pi i \alpha_{T}(m)}, \nu_{T}(m) \cdot e^{2 \pi i \beta_{T}(m)}\right)\right): i=\sqrt{-1} \\
0 \leq \mu_{T}^{q}(m)+\nu_{T}^{q}(m) \leq 1,0 \leq \alpha_{T}^{q}(m)+\beta_{T}^{q}(m) \leq 1, q \in \mathbb{Z}^{+}
\end{array}\right\} .
$$

\section{Complex q-Rung Orthopair Fuzzy Graphs}

As discussed earlier, the notion of CQROFS [14] flexibly described the falsity and truth degrees under uncertainty with the help of complex numbers. In comparison with the CIFS, the CPyFS gives the same results by allowing us wide ground for the selection of degrees of falsity and truth. In this section, we aim to present the notion of CQROFR followed by the conception of CQROFGs and related ideas.

Definition 8. A CQROFR is defined by

$$
R(V, U)=\left\{\left((x, y), \mu_{R}(x, y) e^{i 2 \pi \alpha_{R}(x, y)}, \nu_{R}(x, y) e^{i 2 \pi \beta_{R}(x, y)}\right):(x, y) \in V \times U\right\}
$$

where $\quad 0 \leq \mu_{R}^{q}(x, y)+v_{R}^{q}(x, y) \leq 1 \quad$ and $\quad 0 \leq \alpha_{R}^{q}(x, y)+$ $\beta_{R}^{q}(x, y) \leq 1, q \in \mathbb{Z}^{+}$.

Definition 9. A CQROFG is a pair $G=(C, D)$, where $C$ is a CQROF vertex set on $\mathscr{X}$ and $D$ is a $\mathrm{QROF}$ relation on $\mathscr{X}$ such that

$$
\begin{aligned}
& \mu_{D}(x y) \leq \mu_{C}(x) \wedge \mu_{C}(y), \\
& v_{D}(x y) \leq \nu_{C}(x) \vee \nu_{C}(y), \\
& \alpha_{D}(x y) \leq \alpha_{C}(x) \wedge \alpha_{C}(y), \\
& \beta_{D}(x y) \leq \beta_{C}(x) \vee \beta_{C}(y),
\end{aligned}
$$

and $\quad 0 \leq \mu_{D}^{q}(x y)+v_{D}^{q}(x y) \leq 1, \quad 0 \leq \alpha_{D}^{q}(x y)+\beta_{D}^{q}(x y) \leq$ $1, q \in \mathbb{Z}^{+}$, for all $x, y \in \mathscr{X}$.
Definition 10. A CQROFMS $T$ is characterized in a way where falsity degree $\mathscr{F}_{T}(m)$ and truth degree $\mathrm{T}_{T}(m)$ functions give to every object $\mathscr{F} \in \mathscr{X}$ degrees of truth and falsity in $\mathbb{C}$ by $\mathrm{T}_{T}(m): \mathscr{X} \longrightarrow Q$ and $\mathscr{F} \bigoplus_{T}(m): \mathscr{X} \longrightarrow$ $Q^{\prime}$, that is, $Q=\{c: c \in \mathbb{C},|c| \leq 1\}, Q^{\prime}=\left\{c^{\prime}: c^{\prime} \in \mathbb{C},\left|c^{\prime}\right| \leq 1\right\}$ and $c=\mu_{T}^{p}(m) e^{2 \pi i \alpha_{T}^{p}(m)}$ and $C^{\prime}=v_{T}^{p}(m) e^{2 \pi i \beta_{T}^{p}(m)}$. The elements of $\mathrm{T}_{T}(m)$ i.e. $\mu_{T}^{1}(m) e^{2 \pi \iota \alpha_{T}^{1}(m)}, \mu_{T}^{2} e^{2 \pi \iota \alpha_{T}^{2}(m)}, \ldots, \mu_{T}^{\mathfrak{s}}(m)$ $e^{2 \pi i \alpha_{T}^{\mathfrak{s}}(m)}$, where $\mu_{T}^{1}(m) \geq \mu_{T}^{2}(m) \cdots \geq \mu_{T}^{\mathfrak{s}}(m)$ (for amplitude terms) and $\alpha_{T}^{1}(m) \geq \alpha_{T}^{2}(m) \geq \cdots \geq \alpha_{T}^{\mathfrak{B}}(m)$ (for phase terms). A similar pattern can be followed for the elements of $\mathscr{F} \bigoplus_{T}(m)$ such that $0 \leq\left(\mu_{T}^{R}(m)\right)^{q}+\left(\nu_{T}^{p}(m)\right)^{q} \leq 1$ and $0 \leq\left(\alpha_{T}^{p}(m)\right)^{q}+\left(\beta_{T}^{p}(m)\right)^{q} \leq 1$ for all $p=1,2, \ldots, \mathfrak{s}$. For convenience, we have

$$
\left\{\left(m \cdot \mu_{T}^{p}(m) e^{2 \pi \iota \alpha_{T}^{p}(m)}, v_{T}^{p r e}(m) e^{2 \pi \iota \beta_{T}^{p}(m)}, \mathcal{p}=1,2, \ldots, \mathfrak{g}\right): m \in \mathscr{X}\right\}
$$

Definition 11. Let $T=\left(m, \mu_{T}(m) e^{i \alpha_{T}(m)}, \beta_{T}(m) e^{i \beta_{T}(m)}\right)$ be a CQROFS on $V$, and let $S=\left\{\left(m n, \mu_{S}(m n)_{k} e^{i \alpha_{S}(m n) k}\right.\right.$, $\left.\left.(m n)_{k} \nu_{T} e^{i \beta_{S}(m n) k}\right), k=1,2,3, \ldots, \mathfrak{g}: m n \in V \times V\right\} \quad$ be $\quad$ a CQROFMS on $V \times V$ such that 


$$
\begin{aligned}
\mu_{S}(m n)_{k} & \leq\left(\mu_{T}(m) \wedge \mu_{T}(n)\right), \\
v_{S}(m n)_{k} & \leq\left(v_{T}(m) \vee v_{T}(n)\right), \\
\alpha_{S}(m n)_{k} & \leq\left(\alpha_{T}(m) \wedge \alpha_{T}(n)\right), \\
\beta_{S}(m n)_{k} & \leq\left(\beta_{T}(m) \vee \beta_{T}(n)\right),
\end{aligned}
$$

$\forall, k=1,2,3, \ldots, \mathfrak{g}$.
Then, $G=(T, S)$ is called a CQROFMG.

Example 1. Let $G^{*}=(V, E)$ be an $\mathrm{MG}$, where $V=\left\{v_{1}, v_{2}, v_{3}, v_{4}\right\}$ and $E=\left\{v_{1} v_{2}, v_{2} v_{3}, v_{1} v_{4}, v_{1} v_{4}, v_{3} v_{4}\right\}$. Suppose a CQROF vertex set $A$ and CQROF multiedge set $B$ defined on $V$ as $V \times V$, respectively.

$$
\begin{aligned}
& A=\left(\begin{array}{c}
\left(v_{1}, 0.41 e^{i 2 \pi(0.31)}, 0.69 e^{i 2 \pi(0.34)}\right),\left(v_{2}, 0.21 e^{i 2 \pi(0.26)}, 0.59 e^{i 2 \pi(0.14)}\right), \\
\left(v_{3}, 0.41 e^{i 2 \pi(0.46)}, 0.79 e^{i 2 \pi(0.34)}\right),\left(v_{4}, 0.31 e^{i 2 \pi(0.31)}, 0.79 e^{i 2 \pi(0.14)}\right),
\end{array}\right) \\
& B=\left(\begin{array}{c}
\left(v_{1} v_{2}, 0.16 e^{i 2 \pi(0.14)}, 0.34 e^{i 2 \pi(0.15)}\right),\left(v_{2} v_{3}, 0.21 e^{i 2(0.26)}, 0.79 e^{i 2 \pi(0.34)}\right), \\
\left(v_{3} v_{4}, 0.26 e^{i 2 \pi(0.31)}, 0.69 e^{i 2 \pi(0.34)}\right),\left(v_{1} v_{4}, 0.21 e^{i 2 \pi(0.31)}, 0.74 e^{i 2 \pi(0.34)}\right), \\
\left(v_{1} v_{4}, 0.11 e^{i 2 \pi(0.31)}, 0.69 e^{i 2 \pi(0.34)}\right) .
\end{array}\right)
\end{aligned}
$$

Then, the pair $(A, B)$ forms a CQROFMG and is portrayed in Figure 1.

Definition 12. Let $S=\left\{\left(m n, \mu_{S}(m n)_{k} e^{i \alpha_{S}} \quad(m n)_{k}, v_{S}(m n)_{k}\right.\right.$ $\left.\left.e^{i \beta_{S}(m n)_{k}}\right), k=1,2,3, \ldots, \mathfrak{G}: m n \in V \times V\right\}$ be a CQROF multiedge set in CQROFMG $G$. Then, a strong multiedge $m n$ of $G$ is elaborated by

$$
\begin{aligned}
& \mu_{S}(m n)_{k} \geq \frac{1}{2}\left\{\mu_{T}(m) \wedge \mu_{T}(n)\right\} \\
& \nu_{S}(m n)_{k} \leq \frac{1}{2}\left\{\nu_{T}(m) \vee \nu_{T}(n)\right\} \\
& \alpha_{S}(m n)_{k} \geq \frac{1}{2}\left\{\alpha_{T}(m) \wedge \alpha_{T}(n)\right\} \\
& \beta_{S}(m n)_{k} \leq \frac{1}{2}\left\{\beta_{T}(m) \vee \beta_{T}(n)\right\}, \quad \forall k=1,2,3,4, \ldots, \mathfrak{S} .
\end{aligned}
$$

To illustrate Definition 5, we consider an example based on Figure 1 as follows.

Example 2. By using the information of Example 1, we have $\left(\mu_{S}\left(v_{1} v_{2}\right) e^{i \alpha_{S}\left(v_{1} v_{2}\right)}, v_{S}\left(v_{1} v_{2}\right) e^{i \beta_{S}\left(v_{1} v_{2}\right)}\right)$ as a strong edge because
$0.16 \geq(1 / 2)(0.41 \wedge 0.21), 0.34 \leq(1 / 2)(0.69 \vee 0.59)$ and, on the other hand, $(0.14) \geq(1 / 2)((0.31) \wedge(0.26)),(0.15) \leq(1 /$ 2) $((0.34) \vee(0.14))$.

Definition 13. Let $S=\left\{\left(m n, \mu_{S}(m n)_{k} e^{i} \alpha_{S}(m n)_{k}, v_{S}(m n)_{k}\right.\right.$ $\left.e^{\left.i \beta_{S}(m n)_{k}\right)}, k=1,2,3, \ldots, \mathfrak{s}: m n \in V \times V\right\}$ be a CQROF multiedge set in CQROFMG $G$. An edge $m n$ of $G$ is called effective if the following axioms are satisfied for some fixed $k$ :

$$
\begin{aligned}
\mu_{S}(m n)_{k} & =\mu_{T}(m) \wedge \mu_{T}(n), \\
v_{S}(m n)_{k} & =v_{T}(m) \vee v_{T}(n), \\
\alpha_{S}(m n)_{k} & =\alpha_{T}(m) \wedge \alpha_{T}(n), \\
\beta_{S}(m n)_{k} & =\beta_{T}(m) \vee \beta_{T}(n) .
\end{aligned}
$$

Example 3 supports Definition 13.

Example 3. In Figure $1\left(v_{2} v_{3}, \mu_{S}\left(v_{2} v_{3}\right) e^{i \alpha_{S}\left(v_{2} v_{3}\right)}, v_{S}\left(v_{2} v_{3}\right)\right.$ $\left.e^{i \beta_{S}\left(v_{2} v_{3}\right)}\right)$ is said to be an effective edge as in case of amplitude terms, $0.21=0.41 \wedge 0.21,0.79=0.79 \vee 0.59$ and for phase terms, $(0.26)=(0.46) \wedge(0.26),(0.34)=(0.34) \vee(0.14)$.

Definition 14. Let $\mathrm{G}=(T, S)$ be a CQROFMG and

$$
S=\left\{\left(m n, \mu_{S}(m n)_{k} e^{i \alpha_{S}(m n)_{k}}, v_{S}(m n)_{k} e^{i \beta_{S}(m n)_{k}}\right), \quad k=1,2,3, \ldots, \mathfrak{g}: m n \in V \times V\right\}
$$

be a CQROF multiedge set. Then, a CQROFMG $G$ is known as complete if 


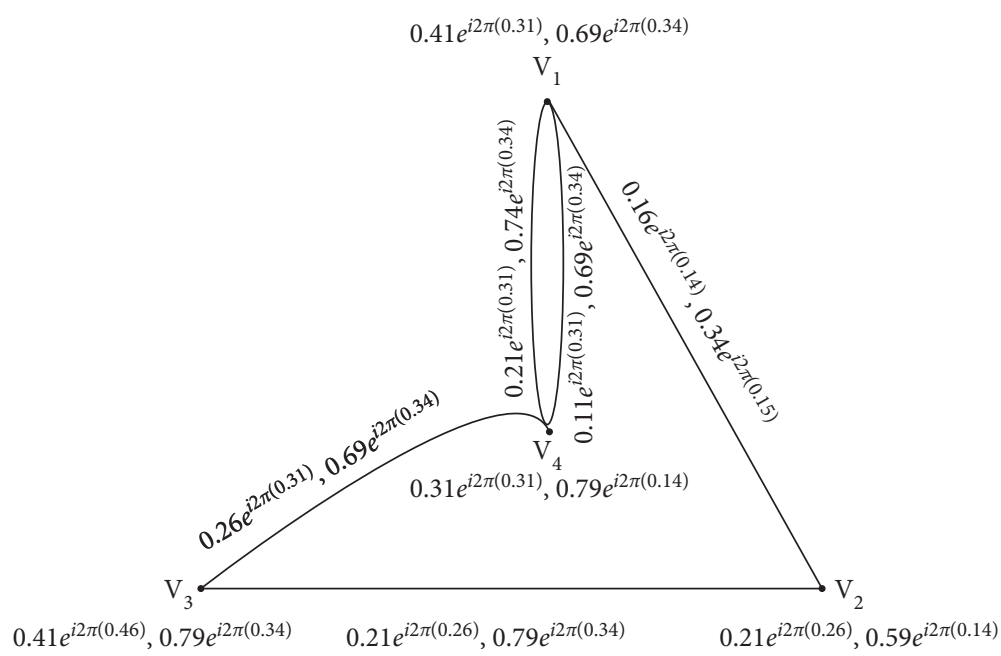

FIgURE 1: Complex q-rung orthopair fuzzy multigraph.

$$
\begin{aligned}
\mu_{S}(m n)_{k} & =\left\{\mu_{T}(m) \wedge \mu_{T}(n)\right\}, \\
v_{S}(m n)_{k} & =\left\{\nu_{T}(m) \vee v_{T}(n)\right\}, \\
\alpha_{S}(m n)_{k} & =\left\{\alpha_{T}(m) \wedge \alpha_{T}(n)\right\}, \\
\beta_{S}(m n)_{k} & =\left\{\beta_{T}(m) \vee \beta_{T}(n)\right\}, \quad \forall k=1,2,3, \ldots, \mathfrak{g}: \forall, m, n \in V .
\end{aligned}
$$

To support Definition 14, we consider Figure 2 followed by Example 4.
Example 4. Consider an $\mathrm{MG} G^{*}=(V, E)$, where $V=\left\{v_{1}, v_{2}, v_{3}, v_{4}\right\} \quad$ and $E=\left\{v_{1} v_{2}, v_{1} v_{3}, v_{1} v_{4}, v_{1} v_{4}, v_{2} v_{3}\right.$, $\left.v_{2} v_{3}, v_{2} v_{4}\right\}$. Suppose a CQROF vertex set $T$ and a CQROF edge set $S$ defined on $V$ and $V \times V$, respectively, as follows:

$$
\begin{aligned}
T= & \left(\begin{array}{c}
\left(v_{1}, 0.41 e^{i 2 \pi(0.21)}, 0.49 e^{i 2 \pi(0.14)}\right),\left(v_{2}, 0.61 e e^{i 2 \pi(0.21)}, 0.69 e^{i 2 \pi(0.24)}\right), \\
\left(v_{3}, 0.71 e^{i 2 \pi(0.26)}, 0.59 e^{i 2 \pi(0.19)}\right),\left(v_{4}, 0.76 e^{i 2 \pi(0.26)}, 0.64 e^{i 2 \pi(0.44)}\right),
\end{array}\right) \\
S= & \left(\begin{array}{c}
\left(v_{1} v_{2}, 0.41 e^{i 2 \pi(0.21)}, 0.69 e^{i 2 \pi(0.24)}\right),\left(v_{1} v_{3}, 0.41 e^{i 2 \pi(0.21)}, 0.59 e^{i 2 \pi(0.21)}\right), \\
\left(v_{1} v_{4}, 0.41 e^{i 2 \pi(0.21)}, 0.64 e^{i 2 \pi(0.44)}\right),\left(v_{1} v_{4}, 0.41 e^{i 2 \pi(0.21)}, 0.64 e^{i 2 \pi(0.44)}\right), \\
\left(v_{2} v_{3}, 0.61 e^{i 2 \pi(0.21)}, 0.69 e^{i 2 \pi(0.24)}\right),\left(v_{2} v_{3}, 0.61 e^{i 2 \pi(0.21)}, 0.69 e^{i 2 \pi(0.24)}\right), \\
\left(v_{2} v_{4}, 0.61 e^{i 2 \pi(0.21)}, 0.69 e^{i 2 \pi(0.24)}\right) .
\end{array}\right)
\end{aligned}
$$

\section{Complex q-Rung Orthopair Fuzzy Planar Graphs}

Planar graphs are those where there is no crossing of edges. Planarity is discussed under uncertainty on a wide range [36]. The aim of this section is to discuss the planarity in the environment of CQROFGs where the truth and falsity degrees of information are flexibly described with a parameter $q$ in a complex plane instead of crisp real values from $[0,1]$.

First, we discuss the notion of strength of CQROF edges and support this concept by Example 5 as follows.

Definition 15. In a CQROFG, the strength of an edge $m n$ is of the form 


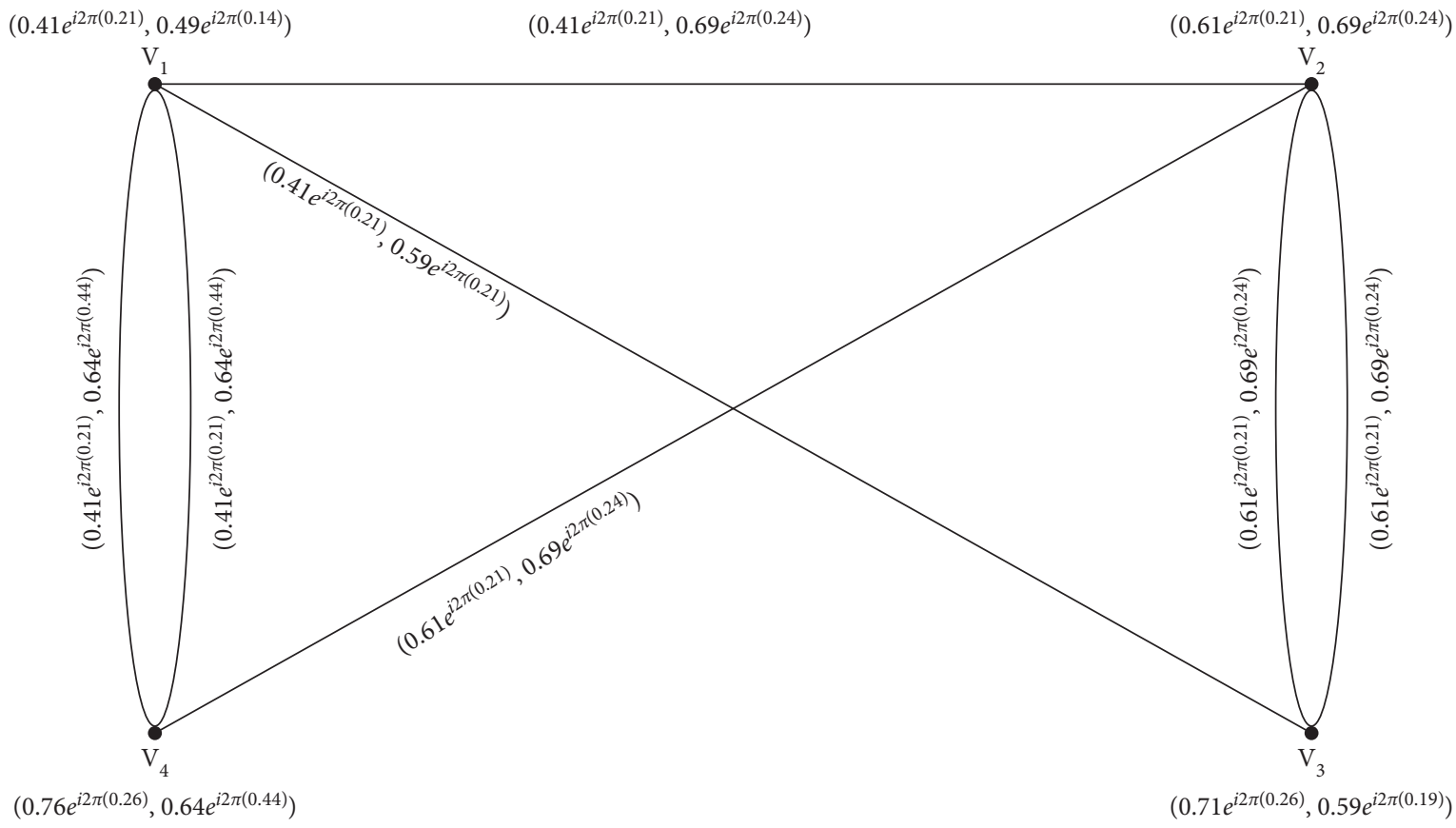

Figure 2: Complete complex q-rung orthopair fuzzy multigraph.

$$
m n=\left(M_{m n}, N_{m n}\right)=\left(\frac{\mu_{S}(m n)_{k} e^{i\left(\alpha_{S}(m n)_{k}\right)}}{\left(\mu_{T}(m) \wedge \mu_{T}(n)\right) e^{i\left(\alpha_{T}(m) \wedge \alpha_{T}(m n)\right)}}, \frac{\nu_{S}(m n)_{k} e^{i\left(\beta_{S}(m n)_{k}\right)}}{\left(\nu_{T}(m) \vee \nu_{T}(n)\right) e^{i\left(\beta_{T}(m) \vee \beta_{T}(n)\right)}}\right) .
$$

If $\quad \mu_{S}(m n) \geq 0.5, \quad(\alpha / 2 \pi) \geq 0.5, \quad v_{S}(m n) \leq 0.5, \quad$ and $(\beta / 2 \pi) \leq 0.5$. Then, the edge $m n$ is known as strong and otherwise weak.
Example 5. Let $G^{*}=(V, E)$ be an $\mathrm{MG}$, where $V=\left\{v_{1}, v_{2}, v_{3}\right\}$ and $E=\left\{v_{1} v_{2}, v_{1} v_{2}, v_{2} v_{3}, v_{2} v_{3}, v_{1} v_{3}\right\}$. Suppose a CQROF vertex set $T$ and CQROF multiedge set $S$ defined on $V$ and $V \times V$, respectively, as follows:

$$
\begin{gathered}
T=\left(\begin{array}{c}
\left(v_{1}, 0.41 e^{i 2 \pi(0.71)}, 0.64 e^{i 2 \pi(0.39)}\right),\left(v_{2}, 0.71 e^{i 2 \pi(0.81)}, 0.34 e^{i 2 \pi(0.59)}\right), \\
\left(v_{3}, 0.26 e^{i 2 \pi(0.10)}, 0.79 e^{i 2 \pi(0.29)}\right),
\end{array}\right) \\
S=\left(\begin{array}{c}
\left(v_{1} v_{2}, 0.41 e^{i 2 \pi(0.91)}, 0.59 e^{i 2 \pi(0.49)}\right),\left(v_{1} v_{2}, 0.41 e^{i 2(0.71)}, 0.29 e^{i 2 \pi(0.59)}\right), \\
\left(v_{2} v_{3}, 0.16 e^{i 2 \pi(0.41)}, 0.79 e^{i 2 \pi(0.49)}\right),\left(v_{2} v_{3}, 0.21 e^{i 2 \pi(0.81)}, 0.74 e^{i 2 \pi(0.59)}\right), \\
\left(v_{1} v_{3}, 0.21 e^{i 2 \pi(0.21)}, 0.29 e^{i 2 \pi(0.39)}\right) .
\end{array}\right)
\end{gathered}
$$

Then, the strength of each edge is computed as follows:

$$
\begin{aligned}
& v_{1} v_{2}=\left(0.1 e^{i 2 \pi(0.64)}, 0.85 e^{i 2 \pi(0.45)}\right), \\
& v_{1} v_{2}=\left(1 e^{i 2 \pi(0.20)}, 0.92 e^{i 2 \pi(0.1)}\right), \\
& v_{2} v_{3}=\left(1 e^{i 2 \pi(0)}, 0.45 e^{i 2 \pi(0.20)}\right), \\
& v_{2} v_{3}=\left(0.81 e^{i 2 \pi(0.71)}, 0.94 e^{i 2 \pi(0.30)}\right), \\
& v_{1} v_{3}=\left(0.81 e^{i 2 \pi(0.11)}, 0.37 e^{i 2 \pi(0.1)}\right) .
\end{aligned}
$$

Since $\quad v_{1} v_{3}=0.81>0.5,(\alpha / 2 \pi) \geq 0.5,0.37<0.5,(\beta$ $/ 2 \pi) \leq 0.5$ ), edge $v_{1} v_{3}$ is the only strong edge in the CQROFMG depicted in Figure 3.

Definition 16. Consider $G=(T, S)$ as a CQROFMG. Let us assume two edges in $S$, i.e., $\left(m n, \mu_{S}(m n)_{p} e^{i \alpha_{S}(m n)_{p}}\right.$, $v_{S}(x y)_{p} e^{\left.i \beta_{S}(m n)_{p}\right)} \quad$ and $\quad\left(x y, \mu_{S}(x y)_{e} e^{i \alpha_{S}(x y)_{0}}, v_{S}(x y)\right.$ $\left.{ }_{a} e^{i \beta_{S}(x y)_{a}}\right)$, intersect at point $O\{\not R, \mathcal{O}=1,2,3, \ldots, \mathfrak{g}\}$. The value of cut point $O$ is given by 


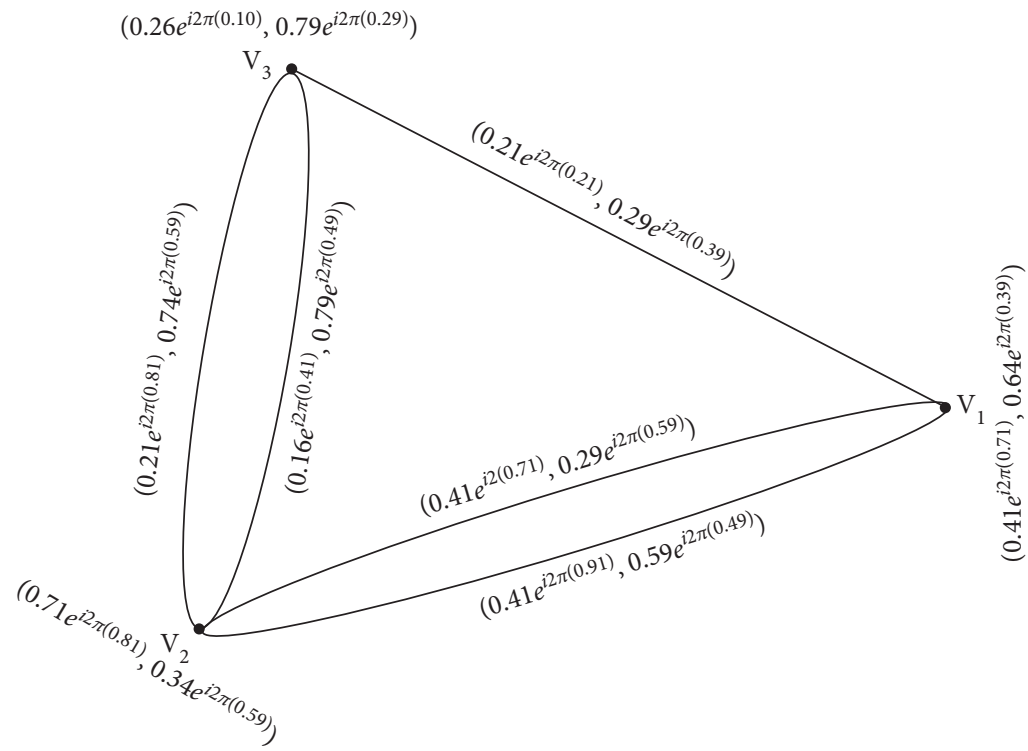

Figure 3: Complex q-rung orthopair fuzzy multigraph.

$$
O=\left(M_{O}, N_{O}\right)=\left(\frac{M_{m n}+M_{m n}}{2}, \frac{N_{m n}+N_{m n}}{2}\right)
$$

where

$$
\begin{aligned}
& \frac{M_{m n}+M_{x y}}{2}=\frac{1}{2}\left(\mu_{m n}+\mu_{x y}-\left(\mu_{m n}\right)\left(\mu_{x y}\right)\right) e^{(i 2 \pi / 2)\left(\left(\alpha_{m n} / 2 \pi\right)+\left(\alpha_{x y} / 2 \pi\right)-\left(\alpha_{m n} / 2 \pi\right)\left(\alpha_{x y} / 2 \pi\right)\right)}, \\
& \frac{N_{m n}+N_{x y}}{2}=\frac{1}{2}\left(\nu_{m n}+\nu_{x y}-\left(\nu_{m n}\right)\left(\nu_{x y}\right)\right) e^{(i 2 \pi / 2)\left(\left(\beta_{m n} / 2 \pi\right)+\left(\beta_{x y} / 2 \pi\right)-\left(\beta_{m n} / 2 \pi\right)\left(\beta_{x y} / 2 \pi\right)\right)} .
\end{aligned}
$$

In the CQROFMG, the cut point $O$ and planarity are inversely proportional, so planarity decreases with the increase in the number of cut points.
Definition 17. Consider $G$ as a CQROFMG. Suppose $\mathrm{O}_{1}, \mathrm{O}_{2}, \ldots, \mathrm{O}_{2}$ to be the cut points of the edges. So, $G$ is called a complex QROF planar graph (CQROFPG) with CQROF PI of $P$ in the form

$$
P=\left(P_{M}, P_{N}\right)=\left(\frac{1}{1+M_{O_{1}}+M_{O_{2}}+\cdots+M_{O_{p}}}, \frac{1}{1+N_{O_{1}}+N_{O_{2}}+\cdots+N_{O_{p}}}\right) .
$$

If there is no cut point in the CQROFPG, then its CQROF PI is assumed as $(1,1)$ and the corresponding crisp graph is a planar graph.

Remark 1. Every CQROFMG with some precise PI is a CQROFPG.
To support the abovementioned remark, we present Example 6.

Example 6. Suppose that $G^{*}=(V, E)$ is an $M G$ in which $V=\left\{v_{1}, v_{2}, v_{3}, v_{4}, v_{5}, v_{6}\right\}$ and $E=\left\{v_{1} v_{2}, v_{1} v_{6}, v_{1} v_{4}, v_{2} v_{5}, v_{2} v_{5}\right.$, $\left.v_{2} v_{3}, v_{3} v_{4}, v_{3} v_{6}, v_{4} v_{5}, v_{6} v_{5}\right\}$ 
Let $T$ and $S$ be the CQROF vertex set and CQROF multiedge set defined on $V$ and $V \times V$, respectively, given as follows by a CQROFMG as depicted in Figure 4:

$$
\begin{gathered}
T=\left(\begin{array}{c}
\left(v_{1}, 0.74 e^{i 2 \pi(0.8)}, 0.46 e^{i 2 \pi(0.31)}\right),\left(v_{2}, 0.59 e^{i 2 \pi(0.19)}, 0.56 e^{i 2 \pi(0.71)}\right), \\
\left(v_{3}, 0.84 e^{i 2 \pi(0.59)}, 0.31 e^{i 2 \pi(0.41)}\right),\left(v_{4}, 0.89 e^{i 2 \pi(0.59)}, 0.41 e^{i 2 \pi(0.61)}\right), \\
\left(v_{5}, 0.44 e^{i 2 \pi(0.39)}, 0.7 e^{i 2 \pi(0.71)}\right),\left(v_{6}, 0.19 e^{i 2 \pi(0.59)}, 0.86 e^{i 2 \pi(0.51)}\right),
\end{array}\right) \\
S=\left(\begin{array}{c}
\left(v_{1} v_{2}, 0.59 e^{i 2 \pi(0.19)}, 0.51 e^{i 2 \pi(0.71)}\right),\left(v_{1} v_{6}, 0.19 e^{i 2 \pi(0.49)}, 0.71 e^{i 2 \pi(0.41)}\right), \\
\left(v_{2} v_{5}, 0.44 e^{i 2 \pi(0.39)}, 0.31 e^{i 2 \pi(0.81)}\right),\left(v_{2} v_{5}, 0.29 e^{i 2 \pi(0.70)}, 0.61 e^{i 2 \pi(0.81)}\right), \\
\left(v_{2} v_{3}, 0.49 e^{i 2 \pi(0.19)}, 0.51 e^{i 2 \pi(0.71)}\right),\left(v_{3} v_{4}, 0.79 e^{i 2 \pi(0.39)}, 0.41 e^{i 2 \pi(0.51)}\right), \\
\left(v_{5} v_{6}, 0.9 e^{i 2 \pi(0.29)}, 0.71 e^{i 2 \pi(0.61)}\right),\left(v_{4} v_{5}, 0.39 e^{i 2 \pi(0.29)}, 0.7 e^{i 2 \pi(0.61)}\right), \\
\left(v_{3} v_{6}, 0.19 e^{i 2 \pi(0.89)}, 0.61 e^{i 2 \pi(0.71)}\right) . \\
P
\end{array}\right)\left(0.99 e^{i 2 \pi(0.38)}, 0.99 e^{i 2 \pi(0.56)}\right) .
\end{gathered}
$$

Let $O_{1}$ and $O_{2}$ be two cut points in the CQROFMG, as shown in Figure 4. For the edges,

$$
\begin{aligned}
& \left(v_{2} v_{5}, 0.44 e^{i 2 \pi(0.39)}, 0.31 e^{i 2 \pi(0.81)}\right), \\
& \left(v_{3} v_{6}, 0.19 e^{i 2 \pi(0.89)}, 0.61 e^{i 2 \pi(0.71)}\right), \\
& \left(v_{2} v_{5}, 0.29 e^{i 2 \pi(0.70)}, 0.61 e^{i 2 \pi(0.81)}\right) .
\end{aligned}
$$

Theorem 1. Let $G$ be a CQROFMG whose intersecting edges are effective. Then, CQROF planarity index $P=\left(P_{M}, P_{N}\right)$ of $G$ is given as

$$
P=\left(P_{M}, P_{N}\right)=\left(\frac{1}{1+m_{O}}, \frac{1}{1+m_{O}}\right),
$$

The strength is

$$
\begin{aligned}
& v_{2} v_{5}=\left(1 e^{i 2 \pi(0)}, 0.44 e^{i 2 \pi(0.1)}\right), \\
& v_{3} v_{6}=\left(1 e^{i 2 \pi(0.30)}, 0.71 e^{i 2 \pi(0.20)}\right), \\
& v_{2} v_{5}=\left(0.66 e^{i 2 \pi(0.32)}, 0.87 e^{i 2 \pi(0.10)}\right) .
\end{aligned}
$$

The first and second cut points are given as follows:

$$
\begin{aligned}
& S_{\mathrm{O}_{1}}=\left(0.49 e^{i 2 \pi(0.37)}, 0.43 e^{i 2 \pi(0.46)}\right), \\
& S_{\mathrm{O}_{2}}=\left(0.49 e^{i 2 \pi(0.37)}, 0.49 e^{i 2 \pi(0.01)}\right) .
\end{aligned}
$$

The PI is given by

$$
\begin{aligned}
m n & =\left(M_{m n}, N_{m n}\right)=\left(\frac{\mu_{S}(m n)_{k} e^{i\left(\alpha_{S}(m n)_{k}\right)}}{\left(\mu_{T}(m) \wedge \mu_{T}(n)\right) e^{i\left(\alpha_{T}(m) \wedge \alpha_{T}(n)\right)}}, \frac{v_{S}(m n)_{k} e^{i\left(\beta_{S}(m n)_{k}\right)}}{\left(\nu_{T}(m) \vee \nu_{T}(n)\right) e^{i\left(\beta_{T}(m) \vee \beta_{T}(n)\right)}}\right), \\
& =\left(\frac{\mu_{S}(m n)_{k} e^{i\left(\alpha_{S}(m n)_{k}\right)}}{\mu_{S}(m n)_{k} e^{i\left(\alpha_{S}(m n)_{k}\right)}} \frac{v_{S}(m n)_{k} e^{i\left(\beta_{S}(m n)_{k}\right)}}{v_{S}(m n)_{k} e^{i\left(\beta_{S}(m n)_{k}\right)}}\right), \\
& =(1,1) .
\end{aligned}
$$

Therefore, the cut point $O_{1}$ of the edges $m n$ and $x y$ is 


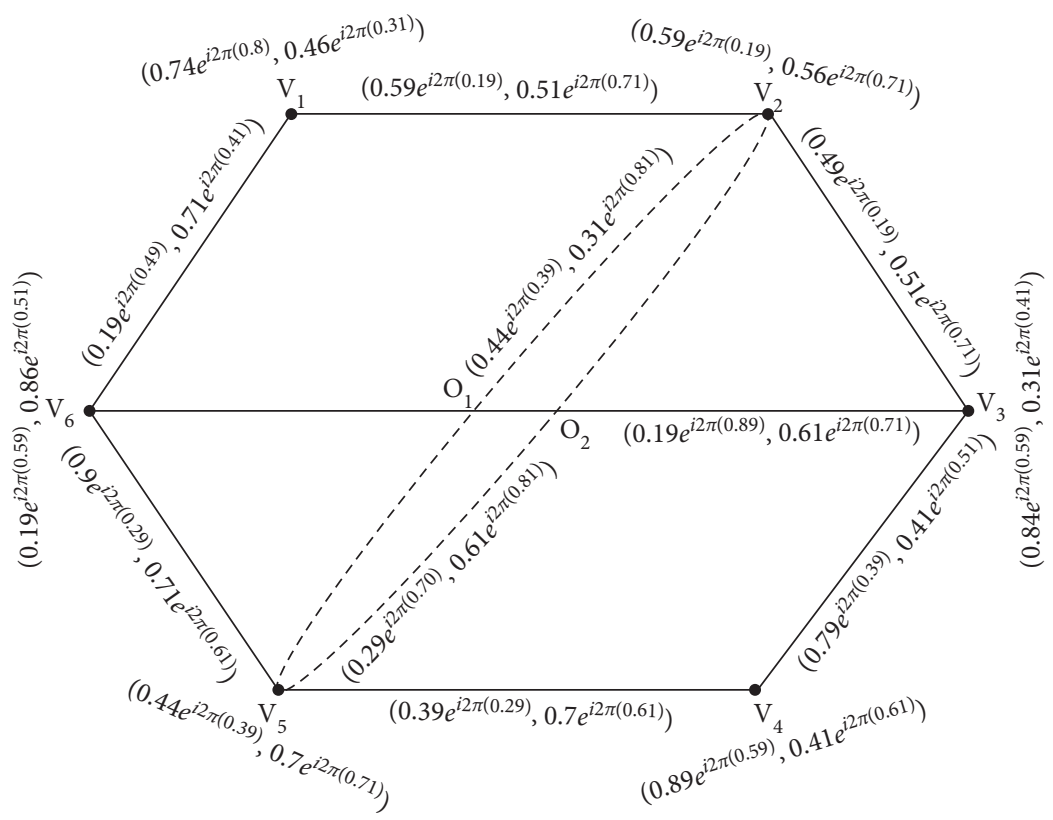

Figure 4: Complex q-rung orthopair fuzzy multigraph.

$$
O_{1}=\left(M_{O_{1}}, N_{O_{1}}\right)=\left(\frac{M_{m n}+M_{x y}}{2}, \frac{N_{m n}+N_{x y}}{2}\right)=\left(\frac{1+1}{2}, \frac{1+1}{2}\right)=(1,1) .
$$

So, $O_{k}=(1,1)$ for $k=1,2,3, \ldots, p$. The PI of the CQROFMG is

$$
\begin{aligned}
P & =\left(P_{M}, P_{N}\right)=\left(\frac{1}{1+\left\{M_{O_{1}}+M_{O_{2}}+\cdots+M_{O_{p}}\right\}}, \frac{1}{1+\left\{N_{O_{1}}+N_{O_{2}}+\cdots+N_{O_{R}}\right\}}\right) \\
& =\left(\frac{1}{1+\{1+1+\cdots+1\}}, \frac{1}{1+\{1+1+\cdots+1\}}\right), \\
& =\left(\frac{1}{1+m_{O}}, \frac{1}{1+m_{O}}\right) .
\end{aligned}
$$

Here, $O=\{1+1+\cdots+1\}$ denotes the number of crossing among the edges in $G$.

\section{Faces and Dual Graphs of CQROFPGs}

Faces in graphs are termed as those regions which are surrounded by edges. The presence of the CQROFF is due to the presence of the edges in CQROFGs. Even the slightest strength CQROF edges guaranteed the existence of CQROFFs. A PI of $\left(1 e^{i(2 \pi)}, 1 e^{i(2 \pi)}\right)$ assures that there is no crossing between the CQQROF edges.

Definition 18. Let a CQROFPG G $=(T, S)$ with PI $(1,1)$ and

$$
S=\left\{\left(m n, \mu_{S}(m n)_{k} e^{i \alpha_{S}(m n)_{k}}, \nu_{S}(m n)_{k} e^{i \beta_{S}(m n)_{k}}\right), k=1,2,3, \ldots, \mathfrak{g}: m n \in V \times V\right\}
$$

A CQROFF is a region bordered by a CQROF edge set $E^{\prime}$ of $E$ of the CQROFPG $G$ defined as 


$$
\begin{array}{ll}
\min \left(\frac{\mu_{S}(m n)_{k}}{\mu_{T}(m) \wedge \mu_{T}(n)}\right) e^{i \min \left(\alpha_{S}(m n)_{k} /\left(\alpha_{T}(m) \wedge \alpha_{T}(n)\right)\right)}, & k=1,2,3, \ldots, \mathfrak{s}: m n \in E^{\prime}, \\
\max \left(\frac{\nu_{S}(m n)_{k}}{\nu_{T}(m) \vee \nu_{T}(n)}\right) e^{i \max \left(\beta_{S}(m n)_{k} /\left(\beta_{T}(m) \vee \beta_{T}(n)\right)\right)}, & k=1,2,3, \ldots, \mathfrak{s}: m n \in E^{\prime} .
\end{array}
$$

Definition 19. A CQROFF is considered a strong CQROFF if $\mu(m n) \geq 0.5, \quad(\alpha / 2 \pi) \geq 0.5, \nu(m n) \leq 0.5$, and $(\beta / 2 \pi) \leq 0.5$ and otherwise weak.

Remark 2. An outer CQROFF is said to possess a limitless region of the CQROFPG; otherwise, it is considered as an inner CQROFF.

Example 7. Suppose that $\mathrm{G}$ is a CQROFPG, as shown in Figure 5. Let $f_{1}, f_{2}$, and $f_{3}$ be the CQROFFs given as follows:

Now, we see that $f_{1}$ is strong and the other two faces $f_{2}$ and $f_{3}$ are weak faces.

$$
\begin{aligned}
& f_{1}=\left(0.51 e^{i 2 \pi(0.81)}, 0.41 e^{i 2 \pi(0.40)}\right), \\
& f_{2}=\left(0.51 e^{i 2 \pi(0.01)}, 0.76 e^{i 2 \pi(0.79)}\right), \\
& f_{3}=\left(0.51 e^{i 2 \pi(0.01)}, 0.99 e^{i 2 \pi(0.79)}\right) .
\end{aligned}
$$

Remark 3. Every strong CQROFF has a strong CQROF edge.

The conception of dual graphs has been widely discussed in the literature, and it has a close connection with planar graphs. There exist some results that relate planarity with duality, i.e., the graphs for which dual graphs exist are known as planar graphs. Due to this reason, we aim to discuss the CQROFDG in our next study and establish its relationship with CQROFPGs.

Definition 20. Consider $\mathrm{G}=(T, S)$ as a $\mathrm{CQROFPG}$ where

$$
S=\left\{\left(m n, \mu_{S}(m n)_{k} e^{i \alpha_{S}(m n)_{k}}, v_{S}(m n)_{k} e^{i \beta_{S}(m n)_{k}}\right), \quad k=1,2,3, \ldots, \mathfrak{g}: m n \in V \times V\right\}
$$

Suppose that the strong CQROFFs of $G$ are $f_{1}, f_{2}, f_{3}, \ldots, f_{3}$. Then, the CQROFDG of $\mathrm{G}$ is a CQROFPG $G^{\prime}=\left(Z^{\prime}, T^{\prime}, S^{\prime}\right)$, where $Z^{\prime}=\left\{s_{k}, k=1,2,3, \ldots\right.$, $p$ \} denotes the nodes of $\mathrm{G}$ for CQROFFs $f_{k}$. Moreover, the degrees of truth and falsity of the vertices are given by mapping $T^{\prime}=\left(\mu_{T^{\prime}} e^{i \alpha_{T^{\prime}}}, v_{T^{\prime}} e^{i \beta_{T^{\prime}}}\right): Z^{\prime} \longrightarrow Q$ such that

$$
\begin{aligned}
& \mu_{T}\left(s_{s}\right)_{k}=\max \left\{\mu_{S}(t s)_{k}\right\}, \\
& \nu_{T^{\prime}}\left(\varsigma_{S^{\prime}}\right)_{k}=\min \left\{v_{S}(t s)_{k}\right\} \text {, } \\
& \alpha_{T^{\prime}}\left(s_{s}\right)_{k}=\max \left\{\alpha_{S}(t s)_{k}\right\} \text {, } \\
& \left.\beta_{T^{\prime}}()_{\zeta}\right)_{k}=\min \left\{\beta_{S}(t s)_{k}\right\} \text {. }
\end{aligned}
$$

The edge $t s$ is near to the strong CQROFF. Between every two CQROFFs $f_{k}$ and $f_{h}$, there may exist more than one common edge. Hence, there may occur more than one edge $\varsigma_{k} \varsigma_{h}$ between two nodes in CQROFDG $G^{\prime}$. The degrees of the truth and falsity of CQROF edges of the CQROFDG can be computed as

$$
\begin{aligned}
& \mu_{S}^{r}(t s)_{\mathfrak{S}}=\mu_{S^{\prime}}\left(s_{k} s_{h}\right)_{r} e^{i \alpha_{s} s^{\prime}\left(s_{\zeta} s_{h}\right)_{r},} \\
& v_{S}^{r}(t s)_{\mathfrak{Z}}=v_{S^{\prime}}\left(s_{k} s_{h}\right)_{r} e^{i \beta_{S^{\prime}}\left(s_{k} \varsigma_{h}\right)_{r},},
\end{aligned}
$$

where $(t s)^{r}$ denotes those edges that lie in the surrounding of strong CQROFFs $f_{h}$ and $f_{k}$ where $r=1,2,3, \ldots, \mathfrak{I}$. As there are no cut points in the CQROFDG $G^{\prime}$ of CQROFPG $\mathrm{G}$, its PI is $(1,1)$.

Example 8. Consider a CQROFPG $G=(\mathscr{Z}, T, S)$ as portrayed in Figure 6 with $\mathscr{Z}=\left\{v_{1}, v_{2}, v_{3}, v_{4}\right\}$. Calculation shows that three strong CQROFFs $f_{1}, f_{2}$, and $f_{3}$ exist whose vertices are shown by little circles and the edges are displayed dotted. The nodes of CQROFDGs $z_{1}, z_{2}, z_{3}$ are computed as follows:

$$
\begin{aligned}
& z_{1}=\left(0.39 e^{i 2 \pi(0.29)}, 0.31 e^{i 2 \pi(0.02)}\right), \\
& z_{2}=\left(0.29 e^{i 2 \pi(0.24)}, 0.31 e^{i 2 \pi(0.16)}\right), \\
& z_{3}=\left(0.39 e^{i 2 \pi(0.29)}, 0.31 e^{i 2 \pi(0.02)}\right) .
\end{aligned}
$$

We observed that $v_{1} v_{3}$ comes out to be the only common edge between $f_{1}$ and $f_{2}$ in $G$. Hence, there exists only one edge between the vertices $z_{1}$ and $z_{2}$ in CQROFDG $G^{\prime}$ of $G$ since $z_{1} z_{2}=v_{1} v_{3}, z_{1} z_{3}=v_{3} v_{4}, z_{1} z_{3}=v_{1} v_{4}, z_{2} z_{3}=v_{2} v_{3}$, and $z_{2} z_{3}=v_{1} v_{2}$

The edge set of the CQROFDG is given by 


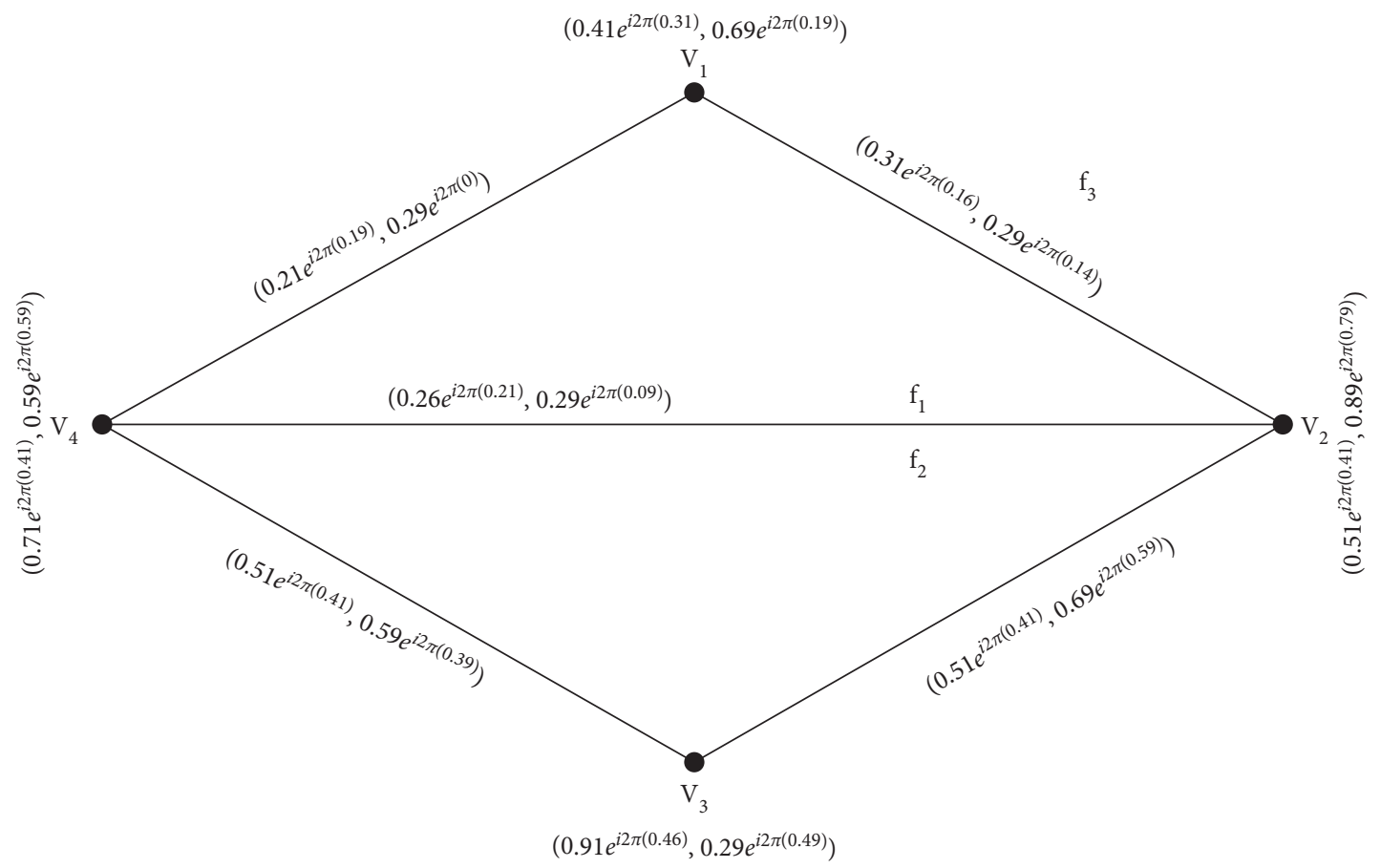

FIgURE 5: The complex q-rung orthopair fuzzy face.

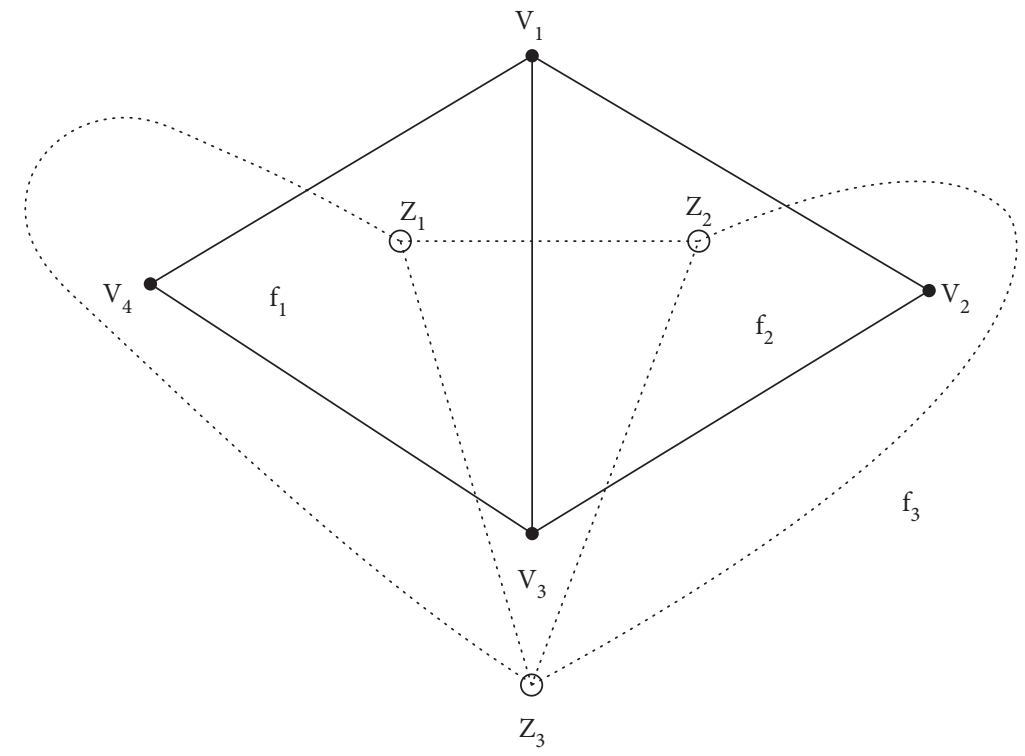

FIgURE 6: Complex q-rung orthopair fuzzy dual graph of the CQROFPG.

$$
S^{\prime}=\left(\begin{array}{c}
\left(z_{1} z_{2}, 0.31 e^{i 2 \pi(0.18)}, 0.29 e^{i 2 \pi(0)}\right),\left(z_{1} z_{3}, 0.41 e^{i 2 \pi(0.31)}, 0.29 e^{i 2 \pi(0)}\right), \\
\left(z_{1} z_{3}, 0.21 e^{i 2 \pi(0.21)}, 0.29 e^{i 2 \pi(0)}\right),\left(z_{2} z_{3}, 0.31 e^{i 2 \pi(0.26)}, 0.29 e^{i 2 \pi(0.14)}\right), \\
\left(z_{2} z_{3}, 0.31 e^{i 2 \pi(0.26)}, 0.29 e^{i 2 \pi(0.14)}\right) .
\end{array}\right)
$$

Hence, $\quad G^{\prime}=\left(Z^{\prime}, T^{\prime}, S^{\prime}\right)$ is a CQROFDG of $G=(\mathscr{Z}, T, S)$.
The dotted line shows a CQROFDG of the CQROFPG which is represented by the bold edges in Figure 6. 
Theorem 2. Let $G$ and $G$ 'denote a CQROFPG with no weak edge and a CQROFDG, respectively, with $v, v^{\prime}$ as their vertices, $f, f^{\prime}$ as their strong faces, and $e, e^{\prime}$ as their edges. Then, $v^{\prime}=f, f^{\prime}=v$, and $e=e^{\prime}$.

Proof. The proof is straightforward.

Theorem 3. Consider a CQROFPG $G$ and its dual graph $G^{\prime}$. Let $f^{\prime}$ and $v$ denote the strong CQROFFs in $G^{\prime}$ and the number of vertices in $G$, respectively. Then, $f \leq v$.

Proof. Let $G^{\prime}$ denote a CQROFDG of a CQROFPG $G$ and $v$ be the vertices in $G$ and $f^{\prime}$ be strong CQROFFs in $G^{\prime}$. As $G$ contains both weak and strong CQROF edges and the CQROFDG does not contain any weak CQROF edge, the existence of some weak CQROF edges in $G$ leads to the fact that some vertices have weak CQROF edges in their neighborhood. Let $x$ denote such vertex, and any strong CQROFF has no vertex like $x$. The remaining vertices are $v-x$ (by excluding these vertices and neighboring edges). Furthermore, $f^{\prime}=v-x$ which means $f^{\prime} \leq v$.

Theorem 4. The absence of a weak edge in a CQROFDG $G$ of a CQROFPG $G$ assures that the degrees of truth and falsity of $C Q R O F$ edges of $G^{\prime}$ and $G$ are equivalent.

Proof. Suppose that $G$ is a CQROFPG having no weak edges. The CQROFDG of $G$ is the family of $G^{\prime}$, and it contained no crossing between any edge. Consider that $f_{1}, f_{2}, f_{3}, \ldots, f_{3}$ are strong CQROFFs of $G$. By using the definition of CQROFDG, the degrees of truth and falsity of CQROF edges of the CQROFDG are given as

$$
\begin{aligned}
& \mu_{S^{\prime}}\left(s_{k} s_{h}\right)_{r}=\mu_{S}^{r}(t s)_{\mathfrak{g}}, \\
& v_{S^{\prime}}\left(s_{k} s_{h}\right)_{r}=v_{S}^{r}(t s)_{\mathfrak{g}}, \\
& \alpha_{S^{\prime}}\left(s_{k} s_{h}\right)_{r}=\alpha_{S}^{r}(t s)_{\mathfrak{g}}, \\
& \beta_{S^{\prime}}\left(s_{k} s_{h}\right)_{r}=\beta_{S}^{r}(t s)_{\mathfrak{g}} .
\end{aligned}
$$

This represents $(t s)^{r}$ is an edge bordered by strong CQROFFs $f_{h}$ and $f_{k}$. The number of common edges in the surrounding between $f_{h}$ and $f_{k}$ is $r$, where $r=1,2, \ldots, \mathfrak{g}$. The two CQROFGs $G$ and $G$ have the same number of CQROF edges, as there are no weak edges in $G$. Hence, there is a CQROF edge in $G^{\prime}$, for every CQROF edge of $G$, with the same degree of truth and falsity.

\section{Isomorphism between Two Complex QROF Planar Graphs}

In this section, we study the isomorphism in CQROFPGs and establish some results to discuss the isomorphic, weak, and coweak isomorphic relations. Each concept is supported by an example.

Definition 21. Let us consider two QROFGs $G_{1}=\left(T_{1}, S_{1}\right)$ and $G_{2}=\left(T_{2}, S_{2}\right)$. A mapping $g: G_{1} \longrightarrow G_{2}$ is called homomorphism defined by $g: V_{1} \longrightarrow V_{2}$ such that (i) $\mu_{T_{1}}(y) \leq \mu_{T_{2}}(g(y)), v_{T_{1}}(y) \geq v_{T_{2}}(g(y))$

(ii) $\mu_{S_{1}}(y z) \leq \mu_{S_{2}}(g(y) g(z)), \nu_{S_{1}}(y z) \geq v_{S_{2}}(g(y) g(z))$, $\forall y \in V_{1}, y z \in E_{1}$

An isomorphic relation between two graphs tells us about the nature of the graph in case we know the properties of one graph. An isomorphism can be established between two CQROFPGs if they have equal division. In our next discussion, we aim to study isomorphisms and their relevant notions in CQROFPGs.

Definition 22. An isomorphism is a bijective mapping $\mathscr{H}: \mathscr{Z}_{1} \longrightarrow \mathscr{Z}_{2}$ of two CQROFPGs $G_{1}$ and $G_{2}$ defined by $\mathscr{H}: G_{1} \longrightarrow G_{2}$ given as

$$
\begin{aligned}
\text { (i) } & \mu_{T_{1}}(m) e^{i \alpha_{T_{1}}(m)}=\mu_{T}(\mathscr{H}(m)) e^{i \alpha_{T_{2}}(\mathscr{H}(m))}, \nu_{T_{1}}(m) e^{i \beta_{T_{1}}(m)} \\
& =v_{T_{2}}(\mathscr{H}(m)) e^{i \beta_{T_{2}}(\mathscr{H}(m))} \\
\text { (ii) } & \mu_{S_{1}}(m n) e^{i \alpha_{S_{1}}(m n)}=\mu_{S_{2}}(\mathscr{H}(m) \mathscr{H}(n)) e^{i \alpha_{S_{2}}(\mathscr{H}(m) \mathscr{H}(n))}, \\
& v_{S_{1}}(m n) e^{i \beta_{S_{1}}(m n)}=v_{S_{2}}(\mathscr{H}(m) \mathscr{H}(n)) e^{i \beta_{S_{2}}(\mathscr{H}(m) \mathscr{H}(n))}, \quad \forall \\
& m \in \mathscr{E}_{1}, m n \in E_{1}
\end{aligned}
$$

To illustrate the isomorphism of CQROFPGs, we consider Example 9.

Example 9. Consider two CQROFPGs $G_{1}=\left(T_{1}, S_{1}\right)$ and $G_{2}=\left(T_{2}, S_{2}\right)$, shown in Figure 7, are isomorphic to each other by the mapping $\mathscr{H}: \mathscr{Z}_{1} \longrightarrow \mathscr{Z}_{2}$ defined by $\mathscr{H}\left(v_{1}\right)=x_{1}, \mathscr{H}\left(v_{2}\right)=x_{2}, \mathscr{H}\left(v_{3}\right)=x_{4}$, and $\mathscr{H}\left(v_{4}\right)=x_{3}$ satisfying the following axioms:

$$
\begin{aligned}
& \mu_{T_{1}}\left(m_{p}\right) e^{i \alpha_{T_{1}}\left(m_{p}\right)}=\mu_{T_{2}}\left(\mathscr{H}\left(m_{p}\right)\right) e^{i \alpha_{T_{2}}\left(\mathscr{H}\left(m_{p}\right)\right)}, \\
& \nu_{T_{1}}\left(m_{p}\right) e^{i \beta_{T_{1}}\left(m_{p}\right)}=\nu_{T_{2}}\left(\mathscr{H}\left(m_{p}\right)\right) e^{i \beta_{T_{2}}\left(\mathscr{H}\left(m_{p}\right)\right)} \text {, } \\
& \mu_{S_{1}}\left(m_{p} n_{t}\right) e^{i \alpha_{S_{1}}\left(m_{p} n_{t}\right)}=\mu_{S_{2}}\left(\mathscr{H}\left(m_{p}\right) \mathscr{H}\left(n_{t}\right)\right) e^{i \alpha_{S_{2}}\left(\mathscr{H}\left(m_{p}\right) \mathscr{H}\left(n_{t}\right)\right)} \text {, }
\end{aligned}
$$

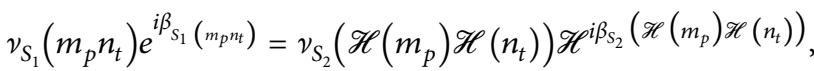

$\forall G_{p} \in \mathbb{Z}_{1}, m_{p} n_{t} \in E_{1}$ where $p, t=1,2,3,4$.

Therefore, $G_{1}$ is isomorphic to $G_{2}$.

Definition 23. A bijective function mapping $\mathscr{H}: \mathscr{Z}_{1} \longrightarrow \mathscr{Z}_{2}$ of two CQROFPGs $G_{1}$ and $G_{2}$ is called weak isomorphism, explained by $\mathscr{H}: G_{1} \longrightarrow G_{2}$, in the following form:

(i) $\mathscr{H}$ is a homomorphism

(ii) $\mu_{T_{T_{1}}}(m) e^{i \alpha_{T_{1}(m)}}=\mu_{T_{2}}(\mathscr{H}(m)) e^{i \alpha_{T_{2}}(\mathscr{H}(m))} \quad \nu_{T_{1}}(m)$
$e^{i \beta_{T_{1}(m)}^{1}}=\nu_{T_{2}}(\mathscr{H}(m)) e^{i \beta_{T_{2}}(\mathscr{H}(m))}, \forall m \in \mathscr{Z}_{1}$

Example 10. Consider two CQROFPGs $G_{1}=\left(T_{1}, S_{1}\right)$ and $G_{2}=\left(T_{2}, S_{2}\right)$, shown in Figure 8, are weak isomorphic to each other by the mapping $\mathscr{H}: \mathscr{Z}_{1} \longrightarrow \mathscr{Z}_{2}$ explained by $\mathscr{H}\left(v_{1}\right)=x_{2}, \mathscr{H}\left(v_{2}\right)=x_{1}, \mathscr{H}\left(v_{3}\right)=x_{5}, \mathscr{H}\left(v_{4}\right)=x_{4}$, and $\mathscr{H}\left(v_{5}\right)=x_{3}$ satisfying the following conditions:

$$
\begin{aligned}
& \mu_{T_{1}}\left(m_{p}\right) e^{i \alpha_{T_{1}}\left(m_{p}\right)}=\mu_{T_{2}}\left(\mathscr{H}\left(m_{p}\right)\right) e^{i \alpha_{T_{2}}\left(\mathscr{H}\left(m_{p}\right)\right)}, \\
& v_{T_{1}}\left(m_{p}\right) e^{i \beta_{T_{1}}\left(m_{p}\right)}=v_{T_{2}}\left(\mathscr{H}\left(m_{p}\right)\right) e^{i \beta_{T_{2}}\left(\mathscr{H}\left(m_{p}\right)\right)},
\end{aligned}
$$




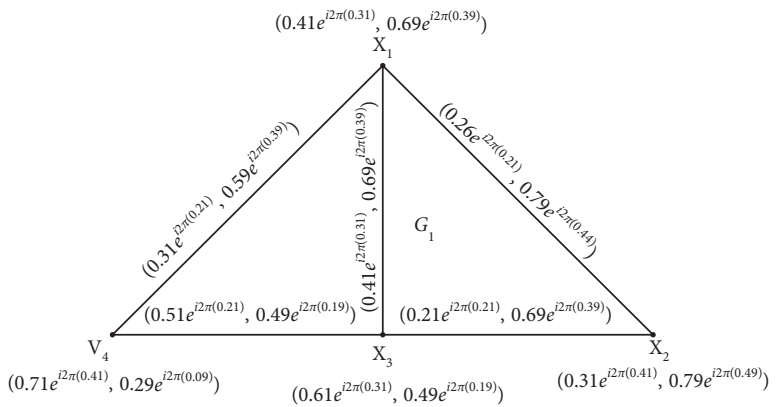

(a)

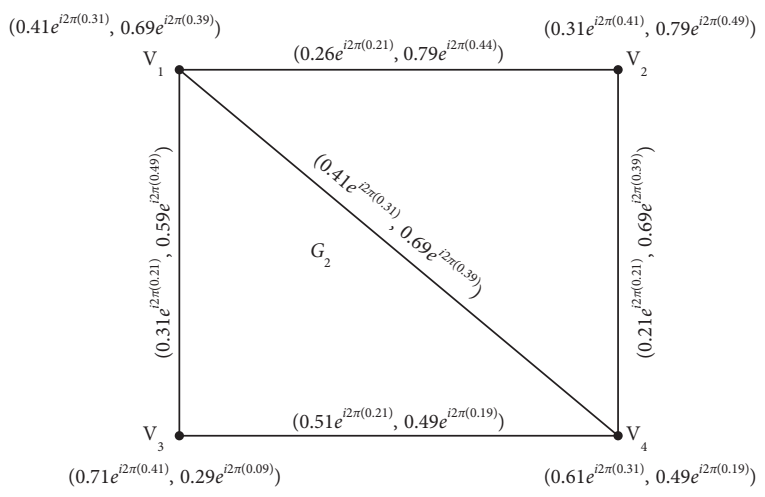

(b)

Figure 7: The two graphs shown are isomorphic.

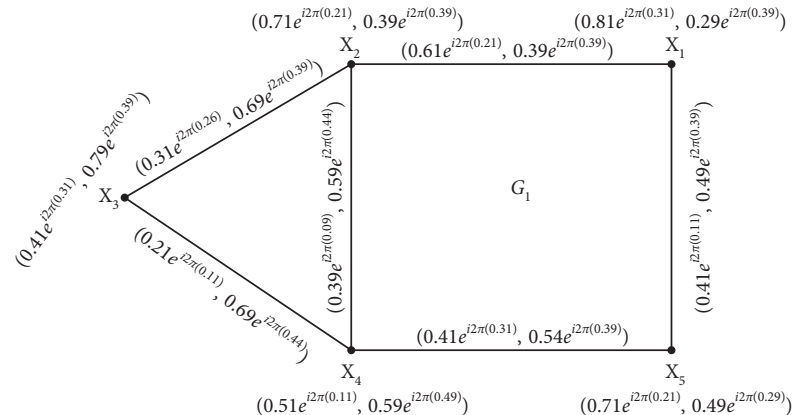

(a)

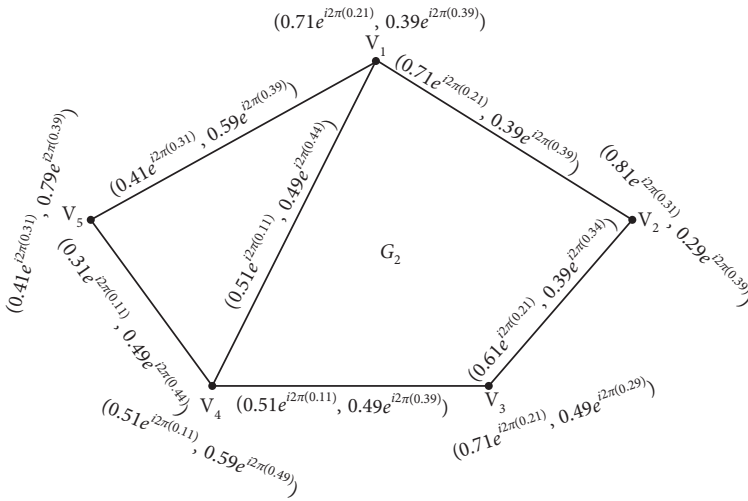

(b)

Figure 8: The two graphs shown are weak isomorphic.

but

$$
\begin{aligned}
& \mu_{S_{1}}\left(m_{p} n_{t}\right) e^{i \alpha_{S_{1}}\left(m_{p} n_{t}\right)} \neq \mu_{S_{2}}\left(\mathscr{H}\left(m_{p}\right) \mathscr{H}\left(n_{t}\right)\right) e^{i \alpha_{S_{2}}\left(\mathscr{H}\left(m_{p}\right) \mathscr{H}\left(n_{t}\right)\right)} \\
& v_{S_{1}}\left(m_{p} n_{t}\right) e^{i \beta_{S_{1}}\left(m_{p} n_{t}\right)} \neq v_{S_{2}}\left(\mathscr{H}\left(m_{p}\right) \mathscr{H}\left(n_{t}\right)\right) e^{i \beta_{S_{2}}\left(\mathscr{H}\left(m_{p}\right) \mathscr{H}\left(n_{t}\right)\right)},
\end{aligned}
$$

$\forall p, t=1,2,3,4,5$. So, there is a weak isomorphism between $G_{1}$ and $G_{2}$.

Definition 24. A bijective mapping $\mathscr{H}: \mathscr{Z}_{1} \longrightarrow \mathscr{Z}_{2}$ of two CQROFPGs $G_{1}$ and $G_{2}$ is called a coweak isomorphism defined by $\mathscr{H}: G_{1} \longrightarrow G_{2}$ as

(i) $\mathscr{H}$ is a homomorphism

(ii) $\mu_{S_{1}}(m n) e^{i \alpha_{S_{1}}(m n)}=\mu_{S_{2}}(\mathscr{H}(m) \mathscr{H}(n)) e^{i \alpha_{S_{2}}(\mathscr{H}(m) \mathscr{H}(n))}$ and $v_{S_{1}}(m n) e^{i \beta_{S_{1}(m n)}}=v_{S_{2}}(\mathscr{H}(m) \mathscr{H}(n))$ $e^{i \beta_{S_{2}}(\mathscr{H}(m) \mathscr{H}(n))} \forall m n \in E_{1}$

Example 11. Two CQROFPGs $G_{1}=\left(T_{1}, S_{1}\right)$ and $G_{2}=\left(T_{2}, S_{2}\right)$, shown in Figure 9, are coweak isomorphic to each other by the mapping $\mathscr{H}: \mathscr{Z}_{1} \longrightarrow \mathscr{Z}_{2}$ defined by
$\mathscr{H}\left(v_{1}\right)=x_{3}, \mathscr{H}\left(v_{2}\right)=x_{1}, \mathscr{H}\left(v_{3}\right)=x_{2}$, and $\mathscr{H}\left(v_{4}\right)=x_{4}$ satisfying the following conditions:

$$
\begin{aligned}
& \mu_{S_{1}}\left(m_{p} n_{t}\right) e^{i \alpha_{S_{1}}\left(m_{p} n_{t}\right)}=\mu_{S_{2}}\left(\mathscr{H}\left(m_{p}\right) \mathscr{H}\left(n_{t}\right)\right) e^{i \alpha_{S_{2}}\left(\mathscr{H}\left(m_{p}\right) \mathscr{H}\left(n_{t}\right)\right)} \\
& \nu_{S_{1}}\left(m_{p} n_{t}\right) e^{i \beta_{S_{1}}\left(m_{p} n_{t}\right)}=v_{S_{2}}\left(\mathscr{H}\left(m_{p}\right) \mathscr{H}\left(n_{t}\right)\right) e^{i \beta_{S_{2}}\left(\mathscr{H}\left(m_{p}\right) \mathscr{H}\left(n_{t}\right)\right)},
\end{aligned}
$$

but

$$
\begin{aligned}
& \mu_{T_{1}}\left(m_{p}\right) e^{i \alpha_{T_{1}}\left(m_{p}\right)} \neq \mu_{T_{2}}\left(\mathscr{H}\left(m_{p}\right)\right) e^{i \alpha_{T_{2}}\left(\mathscr{H}\left(m_{p}\right)\right)}, \\
& \nu_{T_{1}}\left(m_{p}\right) e^{i \beta_{T_{1}}\left(m_{p}\right)} \neq v_{T_{2}}\left(\mathscr{H}\left(m_{p}\right)\right) e^{i \beta_{T_{2}}\left(\mathscr{H}\left(m_{p}\right)\right)},
\end{aligned}
$$

$\forall m_{p} n_{t} \in E_{1}$, where $p, t=1,2,3,4$. So, $G_{1}$ is coweak isomorphic to $G_{2}$.

Theorem 5. If a CQROPG $G$ is isomorphic to a CQROFG $K$ by an isomorphism $\mathscr{H}: G \longrightarrow K$, then $K$ has the same PI as G.

Proof. Let $\mathscr{H}: G \longrightarrow K$ be an isomorphism. Hence, the degrees of truth and falsity of $K$ and $G$ will be equal which establishes a similarity of $K$ with $G$. Hence, the PI and the 


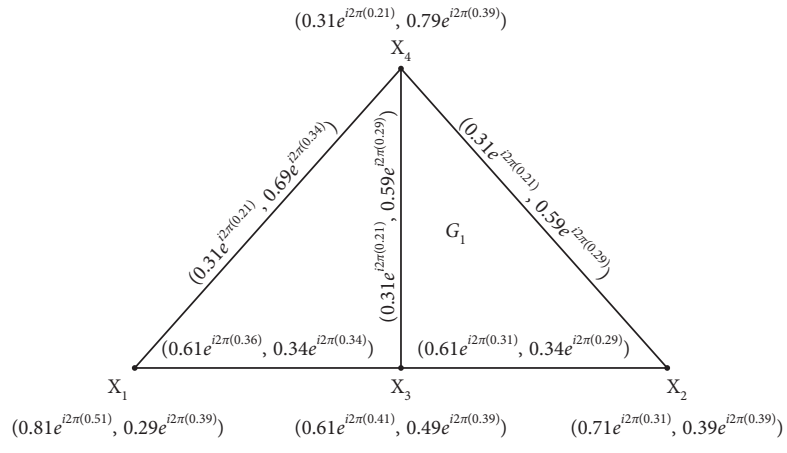

(a)

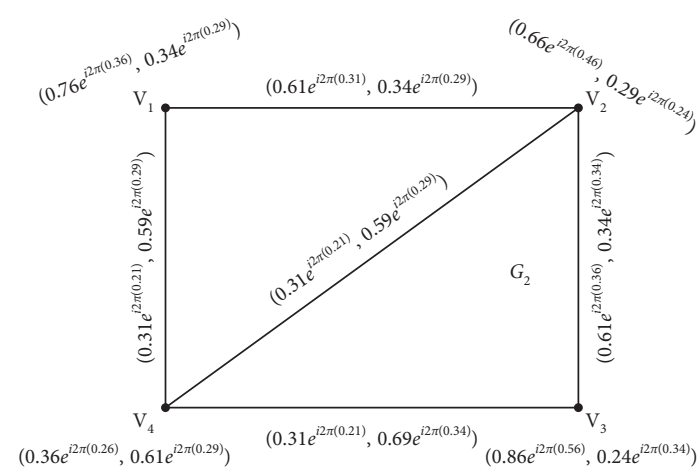

(b)

FIGURE 9: The two graphs shown are coweak isomorphic.

number of cut points between the edges of $K$ and $G$ will be similar. Thus, $K$ is said to have the same PI as $G$.

Theorem 6. Let $P_{1}=\left(P_{M_{1}}, P_{N_{1}}\right)$ and $P_{2}=\left(P_{M_{2}}, P_{N_{2}}\right)$ be the CQROF planarity of two CQROFPGs $G_{1}$ and $G_{2}$, respectively. If $G_{1}$ is weak isomorphic to $G_{2}$, then $\mu_{M_{1}} \geq \mu_{M_{2}}$ and $\nu_{N_{1}} \leq \nu_{N_{2}}$ (for amplitude terms) and $\alpha_{M_{1}} \geq \alpha_{M_{2}}$ and $\beta_{N_{1}} \leq \beta_{N_{2}}$ (for amplitude terms).

Proof. Let $G_{1}$ be weakly isomorphic to $G_{2}$. Then, there exist $\mathscr{H}_{u} \mathscr{H}_{v} \in E_{2}$, for each edge $u v \in E_{1}$. The strength of an edge $m n=\left(M_{m n}, N_{m n}\right)$ is given as

$$
\begin{aligned}
& M_{m n}=\frac{\mu_{S_{1}}(m n) e^{i\left(\alpha_{S_{1}}(m n)_{k}\right)}}{\left(\mu_{T_{1}}(m) \wedge \mu_{T_{1}}(n)\right) e^{i\left(\alpha_{T_{1}}(m) \wedge \alpha_{T_{1}}(n)\right)}}=\mu_{S}(m n) e^{i \alpha_{S}(m n)}, \\
& N_{m n}=\frac{\nu_{S_{1}}(m n)_{k} e^{i\left(\beta_{S_{1}}(m n)_{k}\right)}}{\left(\nu_{T_{1}}(m) \vee v_{T_{1}}(n)\right) e^{i\left(\beta_{T_{1}}(m) \vee \beta_{T_{1}}(n)\right)}}=\nu_{S}(m n) e^{i \alpha_{S}(m n)},
\end{aligned}
$$

$$
\begin{aligned}
M_{\left.\mathscr{H}_{(m)}\right)} \mathscr{\ell}_{(n)}= & \frac{\mu_{S_{2}}\left(\mathscr{H}_{(m)} \mathscr{H}_{(n)}\right)_{k} e^{i \alpha_{S_{2}}\left(\mathscr{H}_{(m)} \mathscr{H}_{(n)}\right)_{k}}}{\left(\mu_{T_{2}}\left(\mathscr{H}_{(m)}\right) \wedge \mu_{T_{2}}\left(\mathscr{H}_{(n)}\right)\right) e^{i\left(\alpha_{T_{2}}\left(\mathscr{H}_{(m)}\right) \wedge \alpha_{T_{2}}\left(\mathscr{H}_{(n)}\right)\right)},} \\
= & \mu_{S}\left(\mathscr{H}_{(m)} \mathscr{H}_{(n)}\right) e^{i_{S_{S}}\left(\mathscr{H}_{(m)} \mathscr{H}_{(n)}\right)} N_{\mathscr{H}_{(m)} \mathscr{H}_{(n)}} \\
= & \frac{\left.v_{S_{2}}\left(\mathscr{H}_{(m)} \mathscr{H}_{(n)}\right)_{k} e^{i\left(\beta_{S_{2}}\left(\mathscr{H}_{(m)} \mathscr{H}_{(n)}\right)\right.}\right)}{\left(v_{T_{2}}\left(\mathscr{H}_{(m)}\right) \vee v_{T_{2}}\left(\mathscr{H}_{(n)}\right)\right) e^{i\left(\beta_{T_{2}}\left(\mathscr{H}_{(m)}\right) \vee \beta_{T_{2}}\left(\mathscr{H}_{(n)}\right)\right)},} \\
= & v_{S}\left(\mathscr{H}_{(m)} \mathscr{H}_{(n)}\right) e^{i \beta_{S}\left(\mathscr{H}_{(m)} \mathscr{H}_{(n)}\right)} .
\end{aligned}
$$

Then, due to weak isomorphism,

$$
\begin{aligned}
& \mu_{S}(m n) \leq \mu_{S}\left(\mathscr{H}_{(m)} \mathscr{H}_{(n)}\right), \\
& v_{S}(m n) \geq v_{S}\left(\mathscr{H}_{(m)} \mathscr{H}_{(n)}\right), \\
& \alpha_{S}(m n) \leq \alpha_{S}\left(\mathscr{H}_{(m)} \mathscr{H}_{(n)}\right), \\
& \beta_{S}(m n) \geq \beta_{S}\left(\mathscr{H}_{(m)} \mathscr{H}_{(n)}\right) .
\end{aligned}
$$

Now, between two edges $m n$ and $x y$, the cut point value $O_{1}=\left(M_{O_{1}}, N_{O_{1}}\right)$ is

$$
\begin{aligned}
& M_{O_{1}}=\frac{1}{2}\left(\mu_{m n}+\mu_{x y}-\left(\mu_{m n}\right)\left(\mu_{x y}\right)\right) e^{(i / 2)\left(\left(\alpha_{m n} / 2 \pi\right)+\left(\alpha_{x y} / 2 \pi\right)-\left(\alpha_{m n} / 2 \pi\right)\left(\alpha_{x y} / 2 \pi\right)\right)}=\mu_{\mathrm{ts}} e^{i \alpha_{t s}}, \quad \text { (say), } \\
& N_{O_{1}}=\frac{1}{2}\left(v_{m n}+v_{x y}-\left(v_{m n}\right)\left(v_{x y}\right)\right) e^{(i / 2)\left(\left(\beta_{m n} / 2 \pi\right)+\left(\beta_{x y} / 2 \pi\right)-\left(\beta_{m n} / 2 \pi\right)\left(\beta_{x y} / 2 \pi\right)\right)}=v_{\mathrm{ts}} e^{i \beta_{t s}}, \quad \text { (say). }
\end{aligned}
$$

Also,

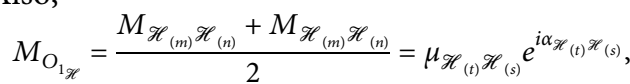

$$
\begin{aligned}
& N_{O_{1 \mathscr{C}}}=\frac{N_{\mathscr{H}_{(m)} \mathscr{H}_{(n)}}+N_{\mathscr{H}_{(m)} \mathscr{H}_{(n)}}}{2}=v_{\mathscr{H}_{(t)} \mathscr{C}_{(s)}} e^{i \beta_{\mathscr{C}_{(t)}} \mathscr{C}_{(s)}} .
\end{aligned}
$$

(i) $\mu_{t s} \leq \mu_{\mathscr{H}_{(t)} \mathscr{H}_{(s)}}, v_{t s} \geq v_{\mathscr{H}_{(t)} \mathscr{H}_{(s)}}$ (for amplitude terms)

(ii) $\alpha_{t s} \leq \alpha_{\mathscr{H}_{(t)} \mathscr{H}_{(s)}}, \beta_{t s} \geq \beta_{\mathscr{H}_{(t)} \mathscr{H}_{(s)}}$ (for phase terms)

In $\mathrm{G}_{2}$, the cut point in the edges $\mathscr{H}(m) \mathscr{H}(n)$ and $\mathscr{H}(x) \mathscr{H}(y)$ is $O_{1_{\mathscr{C}}}$. Now, the $\mathrm{PI}$ is given as 


$$
\begin{aligned}
& \left(P_{M_{1}}, P_{N_{1}}\right)=\left(\frac{1}{1+\left\{M_{O_{1}}+M_{O_{2}}+\cdots+M_{O_{3}}\right\}}, \frac{1}{1+\left\{N_{O_{1}}+N_{O_{2}}+\cdots+N_{O_{3}}\right\}}\right), \\
& =\mu_{M_{1}} e^{i \alpha_{M_{1}}}, \nu_{N_{1}} e^{i \beta_{N_{1}}}
\end{aligned}
$$

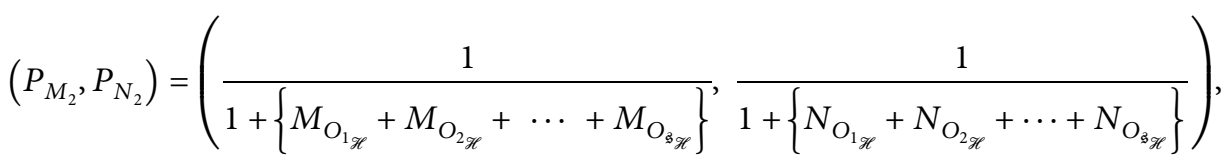

$$
\begin{aligned}
& =\mu_{M_{2}} e^{i \alpha_{M_{2}}}, \nu_{N_{2}} e^{i \beta_{N_{2}}} .
\end{aligned}
$$

Since $G_{1}$ and $G_{2}$ are weak isomorphic to each other, in the certain structural graphical representation of $G_{1}$ and $G_{2}$, the number of cut points $\mathfrak{G}$ is equal. Hence, $\mu_{M_{1}} \geq \mu_{M_{2}}$ and $\nu_{N_{1}} \leq v_{N_{2}}$ (for amplitude terms) and $\alpha_{M_{1}} \geq \alpha_{M_{2}}$ and $\beta_{N_{1}} \leq \beta_{N_{2}}$ (for phase terms).

Theorem 7. Consider $G_{2}$, a CQROFDG of a CQROFDG $G_{1}$, and $G_{1}$ denote a CQROFDG of a CQROFDG G. A coweak isomorphism between $G$ and $G_{2}$ exists if $G$ has no weak edge.

Proof. Consider a CQROPG $G$ with no weak edge in it. Suppose that $G_{1}$ is a CQROFDG of $G$ and $G_{2}$ is a CQROFDG of $G_{1}$. Let $\mathscr{F}_{1}$ denote the strong CQROFFs of $G_{1}$ and $v_{2}$ and $v$ denote the number of vertices of $G_{2}$ and $G$. Then, $\mathscr{F}_{1}=v_{2}$ and $\mathscr{F}_{1}=v$ imply that $v_{2}=v$. Furthermore, by the definition of duality, the truth and falsity degrees of an edge in CQROFDG and CQROFPG are equal. So, a coweak isomorphism between $G$ and $G_{2}$ exists. 7.

Example 12 is given to support the result in Theorem

Example 12. In this example, the CQROFDGs $G, G_{1}$, and $G_{2}$ are depicted as in Figures 10-12, respectively. These CQROFGs shown verify the result discussed in Theorem 7 and establish a coweak isomorphism between $G_{2}$ and $G$.

\section{Application}

In this section, we present an application of the CQROFPGs in electric networks to reduce the short circuits. We used the idea of CQROFPGs to analyze the short-circuit problem based on the planarity of the graph network. We assumed a problem of electricity supply from grid stations using CQROFPGs given below.

Grid stations are the main resources of power supply to our houses that use several lines (known as power lines) that are present throughout our city and streets. The supply lines carry a huge amount of voltage, and to convert it to low voltage, the transformers are used. The transformers consist of various units which further consist of small, tiny wires responsible for the carriage of current. During current flow, sometimes a crossing occurs between the small wires while connecting various units. In a very limited time duration, often the voltage becomes so high that leads to short circuits that are very harmful to the electrical appliances in our houses. The short circuits can be reduced by arranging the wires in a way where the number of crossing needs to be less enough. We use the idea of CQROFPGs to deal with such problems for reducing the number of crossing. A brief algorithm for reducing the edge crossing in the setting of CQROFPGs followed by practical Example 13 is given below.

7.1. Algorithm. This algorithm is to reduce the edge-crossing between nodes. The algorithms involve information in the form of CQROFPGs and use to the concept of strength, values of cut points, and PI in a network. The steps of the algorithm are as follows:

(i) Let the units (nodes) be denoted by $V=\left\{V_{1}, V_{2}, V_{3}, \ldots, V_{3}\right\}$ and the connection (edges) be denoted by $E=\left\{E_{1}, E_{2}, E_{3}, \ldots, E_{3}\right\}$ and $O=\left\{L_{1}, L_{2}, L_{3}, \ldots, L_{\mathfrak{3}}\right\}$ denote the cut points

(ii) This step involves the computation of the strength of edges $E_{\mathfrak{Z}}$ i.e., $\mathfrak{g}=1,2,3, \ldots, p \cdot E_{\mathfrak{B}}=\left(\left(\mu_{S}\left(E_{\mathfrak{B}}\right)_{p}\right.\right.$

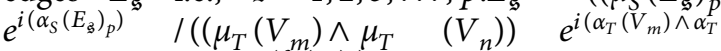
$\left.\left.\left.\left(V_{n}\right)\right)\right)\right) .\left(v_{S}\left(E_{\mathfrak{\xi}}\right)_{p} e^{i\left(\beta_{S}\left(E_{\xi}\right)_{p}\right) /}\left(\left(\nu_{T}\left(V_{m}\right) \vee \quad v_{T}\left(V_{n}\right)\right) e^{i}\right.\right.$ $\left.\left.\left.\left(\beta_{T}\left(V_{m}\right) \vee \beta_{T}\left(V_{n}\right)\right)\right)\right)\right) E_{3}=\left(M_{E_{\xi}}, N_{E_{\xi}}\right)$

(iii) This involves the computation of junction points $L_{p}$ between edges $E_{j} \cdot L_{p}=\left(M_{L_{p}}=(1 / 2)\left(\mu_{E_{m}}+\mu_{E_{n}}-\right.\right.$ $\left.\left(\mu_{E_{m}}\right)\left(\mu_{E_{n}}\right)\right) e^{(i / 2)\left(\left(\alpha_{E_{m}} / 2 \pi\right)+\left(\alpha_{E_{n}} / 2 \pi\right)-\left(\alpha_{E_{m}} / 2 \pi\right)\left(\alpha_{E_{n}} / 2 \pi\right)\right)} N_{L_{p}}=$ $\left.(1 / 2)\left(\nu_{E_{m}}+v_{E_{n}}-\left(\nu_{E_{m}}\right)\left(\nu_{E_{n}}\right)\right) e^{(i /} 2\right)\left(\left(\beta_{E_{m}} / 2 \pi\right)+\left(\beta_{E_{n}} /\right.\right.$ $\left.\left.2 \pi)-\left(\beta_{E_{m}} / 2 \pi\right)\left(\beta_{E_{n}} / 2 \pi\right)\right)\right)$, where $p=1,2,3, \ldots, \mathfrak{Z}$ and $E_{m}, E_{n} \in E$

(iv) evaluate the planarity value using $P=\left(P_{M}, P_{N}\right)=$ $\left(1 /\left(1+\left\{M_{L_{1}}+M_{L_{2}}+\cdots+M_{L_{p}}\right\}\right), 1 /\left(1+\left\{N_{L_{1}}+\right.\right.\right.$ $\left.\left.\left.N_{L_{2}}+\cdots+N_{L_{p}}\right\}\right)\right)$

(v) If $E_{m}$ and $E_{n}$ give no crossing, then we keep the formation of such edges as it is; otherwise, we alter the representation of such edges until the crossing is reduced

(vi) By changing the layout of the $E_{m}$ and $E_{n}$, the intersection becomes minimum and the PI gets maximized 


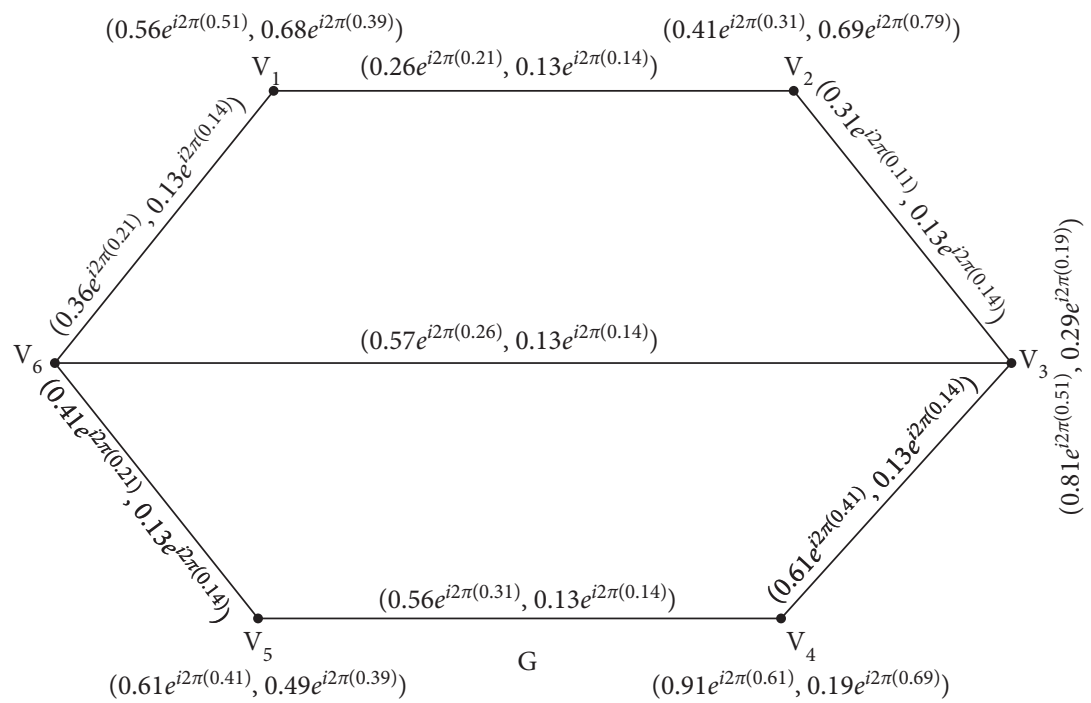

FIGURE 10: Complex QROF planar graph without weak edges.

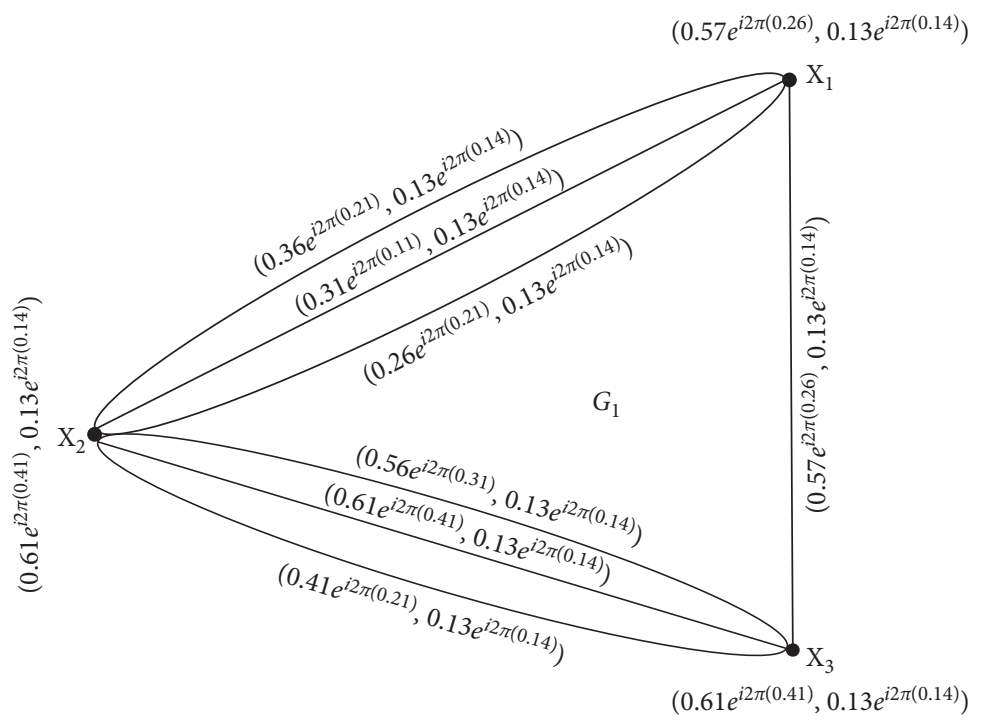

Figure 11: Complex q-rung orthopair fuzzy dual graph $G_{1}$.

Example 13. Let us assume a layout of units in the transformer, as depicted in Figure 13. The nodes represent units $V_{1}, V_{2}, V_{3}, \ldots V_{7}$, and the connections between units are represented by edges, as shown in Figure 13.

At the unit $V_{1}$, we assume that the chances for a low spark are $61 \%$ and those for a high spark are $69 \%$. Similarly, in the case of phase terms, suppose that, in a specific time, the chances for a low spark are $41 \%$ and those for a high spark are $49 \%$. This can be viewed as

$$
\left\{V_{1},\left(0.61 e^{i 2 \pi(0.41)}, 0.69 e^{i 2 \pi(0.49)}\right)\right\} .
$$

For the rest of the nodes, we have

$$
\begin{aligned}
& \left\{V_{2},\left(0.51 e^{i 2 \pi(0.41)}, 0.69 e^{i 2 \pi(0.59)}\right)\right\}, \\
& \left\{V_{3},\left(0.81 e^{i 2 \pi(0.71)}, 0.59 e^{i 2 \pi(0.59)}\right)\right\}, \\
& \left\{V_{4},\left(0.81 e^{i 2 \pi(0.71)}, 0.59 e^{i 2 \pi(0.59)}\right)\right\}, \\
& \left\{V_{5},\left(0.71 e^{i 2 \pi(0.61)}, 0.29 e^{i 2 \pi(0.39)}\right)\right\}, \\
& \left\{V_{6},\left(0.51 e^{i 2 \pi(0.41)}, 0.59 e^{i 2 \pi(0.29)}\right)\right\}, \\
& \left\{V_{7},\left(0.41 e^{i 2 \pi(0.11)}, 0.69 e^{i 2 \pi(0.29)}\right)\right\} .
\end{aligned}
$$

Now, if there is a connection between the units $V_{1}$ and $V_{2}$, then it will be presented by an edge $V_{1} V_{2}$ of the vertices $V_{1}$ and $V_{2} . V_{1} V_{2}$ is given as $\left\{V_{1} V_{2},\left(0.41 e^{i 2 \pi(0.31)}\right.\right.$, 


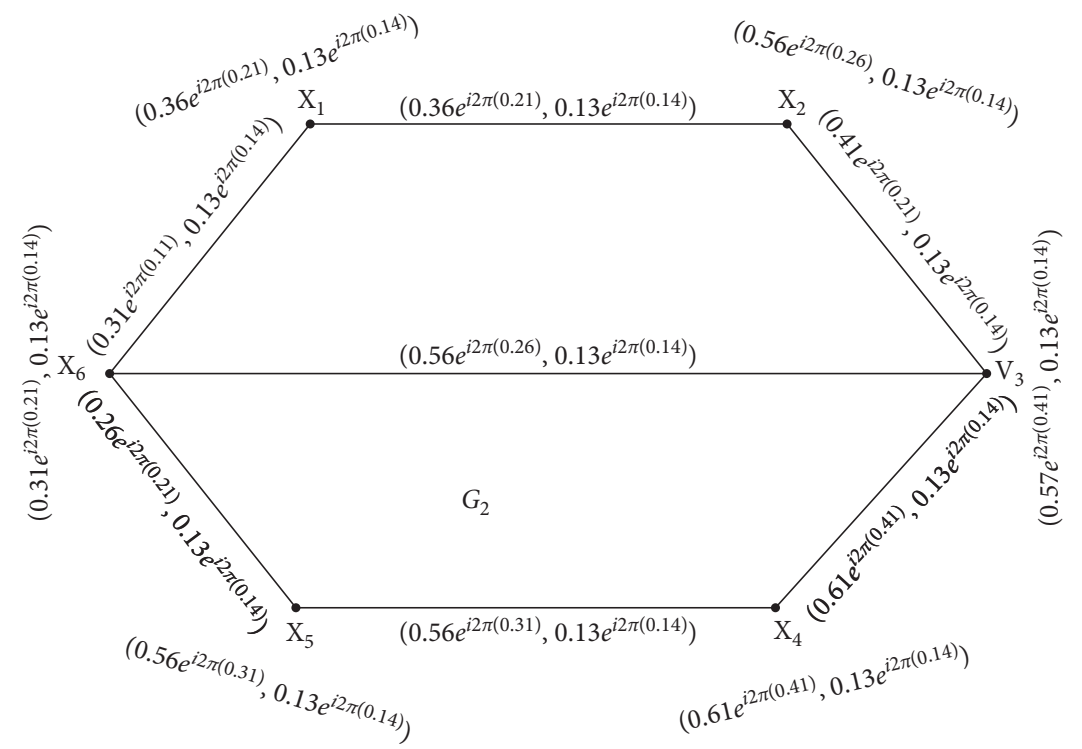

Figure 12: Complex q-rung orthopair fuzzy dual graph $G_{2}$.

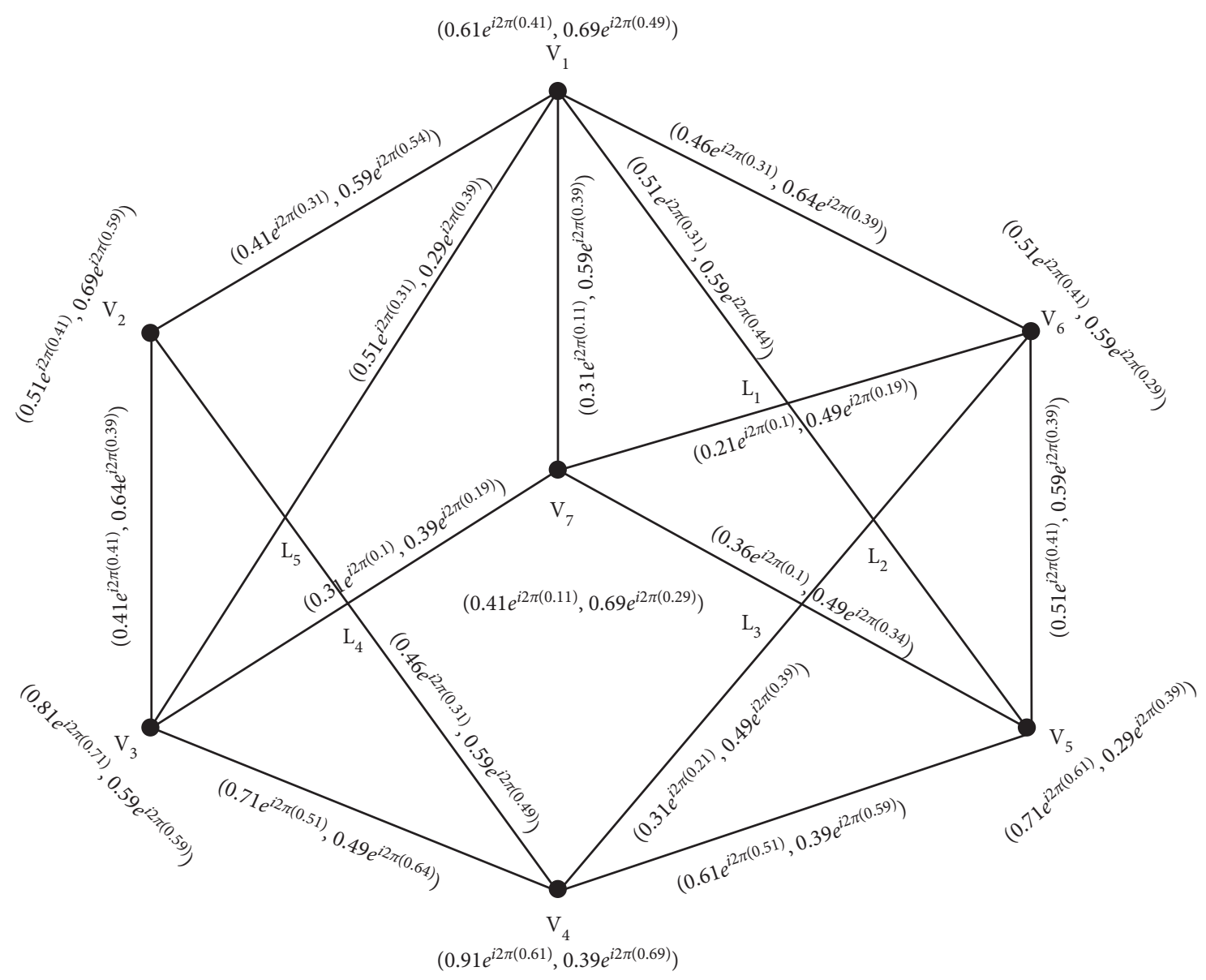

FIGURE 13: Unit connection in the transformer. 


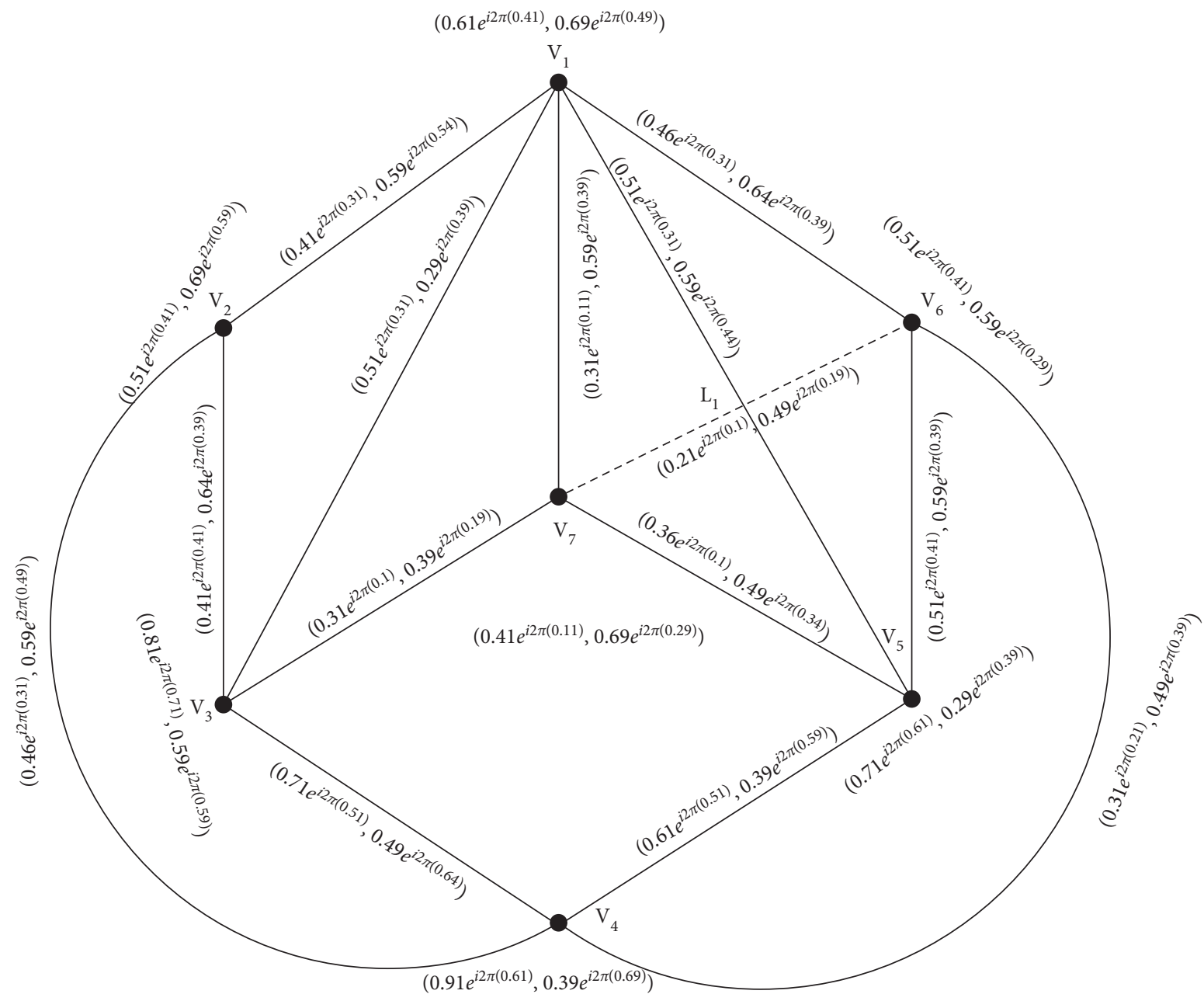

Figure 14: Complex q-rung orthopair fuzzy electric connection model.

$\left.\left.0.59 e^{i 2 \pi(0.54)}\right)\right\}$. The rest of the edges are similarly portrayed in Figure 13. In an edge $\left\{V_{1} V_{2},\left(0.41 e^{i 2 \pi(0.31)}, 0.59 e^{i 2 \pi(0.54)}\right)\right\}$, the chances for a low spark are $41 \%$ and $59 \%$ for a high spark denoted by both amplitude terms of the truth and falsity degrees. Similarly, the phase terms of $31 \%$ and $54 \%$ show the chances for minimum and maximum destruction, respectively. A direct proportion exists between the rates of destruction and the number of cut points. We measure PI for further analysis. The number of cut points between the pair of wires $\left(V_{1} V_{5}, V_{6} V_{7}\right)$, $\left(V_{1} V_{5}, V_{4} V_{6}\right), \quad\left(V_{4} V_{6}, V_{5} V_{7}\right), \quad\left(V_{2} V_{4}, V_{3} V_{7}\right), \quad$ and $\left(V_{1} V_{3}, V_{2} V_{4}\right)$ is denoted by $L_{1}, L_{2}, L_{3}, L_{4}$, and $L_{5}$, respectively. The strengths of wires are

$$
\begin{aligned}
& V 1 V 5=\left(0.84 e^{i 2 \pi(0.76)}, 0.86 e^{i 2 \pi(0.90)}\right), \\
& V 6 V 7=\left(0.51 e^{i 2 \pi(.90)}, 0.71 e^{i 2 \pi(0.66)}\right), \\
& V 4 V 6=\left(0.61 e^{i 2 \pi(0.51)}, 0.83 e^{i 2 \pi(0.57)}\right), \\
& V 5 V 7=\left(0.88 e^{i 2 \pi(.91)}, 0.71 e^{i 2 \pi(0.87)}\right), \\
& V 2 V 4=\left(0.90 e^{i 2 \pi(0.76)}, 0.86 e^{i 2 \pi(0.71)}\right), \\
& V 3 V 7=\left(0.76 e^{i 2 \pi(0.91)}, 0.57 e^{i 2 \pi(0.32)}\right), \\
& V 1 V 3=\left(0.83 e^{i 2 \pi(0.76)}, 0.42 e^{i 2 \pi(0.66)}\right) .
\end{aligned}
$$


The values of cut points are

$$
\begin{aligned}
& L 1=\left(0.68 e^{i 2 \pi(0.83)}, 0.79 e^{i 2 \pi(0.78)}\right), \\
& L 2=\left(0.73 e^{i 2 \pi(0.64)}, 0.85 e^{i 2 \pi(0.74)}\right), \\
& L 3=\left(0.75 e^{i 2 \pi(0.71)}, 0.77 e^{i 2 \pi(0.72)}\right), \\
& L 4=\left(0.83 e^{i 2 \pi(0.84)}, 0.72 e^{i 2 \pi(0.52)}\right), \\
& L 5=\left(0.86 e^{i 2 \pi(0.76)}, 0.64 e^{i 2 \pi(0.69)}\right) .
\end{aligned}
$$

Thus, CQROF PI is $P=\left(0.20 e^{i 2 \pi(0.21)}, 0.21 e^{i 2 \pi(0.22)}\right)$. A low PI shows a high rate of destruction. To lower the crossings, a modified CQROFG is developed in Figure 14. As an inverse relationship exists between the PI and the number of intersections which means that reducing the intersection points increases the PI $P=\left(0.60 e^{i 2 \pi(0.55)}, 0.56 e^{i 2 \pi(0.56)}\right)$ and decreases the destruction rate, there is only one crossing $L_{1}$ in Figure 14 which is irreducible. Therefore, a reduction in the destruction rate and chances of the electric sparks can be achieved by using reasonable quality wires $V_{1}, V_{5}, V_{6}$, and $V_{7}$ and a clearer connection between components in the transformer. This will make the crossing $L_{1}$ less damaging. This concludes that, for lower destruction that can occur at any time, an electrical model based on CQROF information can be adapted.

\section{Comparative Study}

In this segment, we illustrate a comparison of the results discussed in this paper with those already established. A brief comparative analysis of the current work with previous studies is discussed pointwise as follows:

(1) The work in $[36,38]$ discusses only one aspect of the vague information with the help of truth degree which leads to the loss of information as the falsity degree is ignored and results might not be effective.

(2) The concept of planarity discussed in [39] illustrates PI in an uncertain environment with the help of truth and falsity degrees of an IFS which have certain restraints on the falsity and truth degrees of the objects and, hence, cannot be used smoothly.

(3) The PI and its relevant theories and application in the setting of PyFG are discussed in [40] where a larger space for assigning the falsity and truth degrees is provided, but still, some have limitations when it comes to defining nodes and edges under some specific restraints of PyFGs.

(4) Complex falsity and truth degrees are adapted to analyze the theory and results based on PI of CPyFGs. Application of the PI of the CPyFG is also analyzed in [41]. The work in [41] is considered to be more general than the work in $[36,38,39]$. In the setting of CPyFGs, one has an edge by describing the uncertain information with the help of two complex membership grades, but it has restraint on the grades due to which it becomes inapplicable in some cases.

(5) The current study involves complex truth and falsity degrees associated with a variable parameter $q$ that only produces flexibility in taking the complex truth and falsity degrees but can describe the opinion with the help of two kinds of grades that enhance the representation of the uncertain information.

\section{Conclusions}

In this script, we presented a theory related to the CQROFPG keeping in mind its usefulness. We discussed planarity in the setting of CQROFGs where an example is given to support each concept. An algorithm to study a short-circuit problem is developed. A summary of our research is given as follows:

(1) We developed the notions of CQROFR, which leads to the concept of CQROFG. We also introduced the concept of CQROFMS which leads to the concept of CQROFMG. Some examples are given to support the theory.

(2) We introduced the planar graphs in the environment of CQROFGs by introducing the notion of CQROFPGs. The notion of strength and PI is developed and exemplified. The concepts of effective edges are described along with related results.

(3) We extended our study to develop the notion of faces and dual graphs in the environment of CQROFPGs by developing the concepts of CQROFDGs and CQROFFs. An analysis of the strength and weaknesses of the faces is set up. The proposed concepts are supported with examples.

(4) We further establish a comprehensive study on isomorphism in the context of CQROFPGs and discussed the notions of coweek and weak isomorphisms. A few results on the isomorphisms of the CQROFPGs are also investigated.

(5) An application of the CQROFPGs in solving a shortcircuit problem is investigated to see the effectiveness of the proposed work. A practical example is taken to illustrate the applicability of the newly established results in short-circuit problems.

(6) A comparative investigation of the current work is set up with previously established results to show the superiority of the current work.

(7) In the near future, we aim to extend our study of CQROFGs by defining several other relevant terms and notions and to study the applicability of the study in shortest path problems, social network problems, etc. This study when extended to the frame of T-spherical fuzzy graphs $[43,44]$ can give us flexible and more accurate results. 


\section{Data Availability}

No data were used to support this study.

\section{Conflicts of Interest}

The authors declare no conflicts of interest about the publication of this research article.

\section{Acknowledgments}

The authors are grateful to the Deanship of Scientific Research, King Saud University, for funding through Vice Deanship of Scientific Research Chairs.

\section{References}

[1] K. T. Atanassov, "Intuitionistic fuzzy sets," Fuzzy Sets and Systems, vol. 20, no. 1, pp. 87-96, 1986.

[2] L. A. Zadeh, "Fuzzy sets," Information and Control, vol. 8, no. 3, pp. 338-353, 1965.

[3] R. R. Yager, "q- fuzzy subsets," in Proceedings of the Joint IFSA World Congress and NAFIPS Annual Meeting (IFSA/NAFIPS) 2013, pp. 57-61, Edmonton, Alberta, Canada, June 2013.

[4] R. R. Yager, "Generalized orthopair fuzzy sets," IEEE Transactions on Fuzzy Systems, vol. 25, no. 5, pp. 1222-1230, 2017.

[5] M. I. Ali, "Another view on q-Rung orthopair fuzzy sets," International Journal of Intelligent Systems, vol. 33, no. 11, pp. 2139-2153, 2018.

[6] P. Liu and P. Wang, "Some q-Rung orthopair fuzzy aggregation operators and their applications to multiple-attribute decision making," International Journal of Intelligent Systems, vol. 33, no. 2, pp. 259-280, 2018.

[7] P. Wang, J. Wang, G. Wei, and C. Wei, "Similarity measures of q-rung orthopair fuzzy sets based on cosine function and their applications," Mathematics, vol. 7, no. 4, p. 340, 2019.

[8] G. Wei, H. Gao, and Y. Wei, "Some q-Rung orthopair fuzzy Heronian mean operators in multiple attribute decision making," International Journal of Intelligent Systems, vol. 33, no. 7, pp. 1426-1458, 2018.

[9] M. Riaz and M. R. Hashmi, "Linear Diophantine fuzzy set and its applications towards multi-attribute decision-making problems," Journal of Intelligent \& Fuzzy Systems, vol. 37, no. 4, pp. 5417-5439, 2019.

[10] M. Riaz, N. Çağman, I. Zareef, and M. Aslam, "N-soft topology and its applications to multi-criteria group decision making," Journal of Intelligent \& Fuzzy Systems, vol. 36, no. 6, pp. 6521-6536, 2019.

[11] D. Ramot, R. Milo, M. Friedman, and A. Kandel, "Complex fuzzy sets," IEEE Transactions on Fuzzy Systems, vol. 10, no. 2, pp. 171-186, 2002.

[12] A. M. D. J. S. Alkouri and A. R. Salleh, "Complex intuitionistic fuzzy sets," AIP Conference Proceedings, vol. 1482, no. 1, pp. 464-470, 2012.

[13] K. Ullah, T. Mahmood, Z. Ali, and N. Jan, "On some distance measures of complex Pythagorean fuzzy sets and their applications in pattern recognition," Complex \& Intelligent Systems, vol. 6, no. 1, pp. 15-27, 2020.

[14] P. Liu, T. Mahmood, and Z. Ali, "Complex q-Rung orthopair fuzzy aggregation operators and their applications in multiattribute group decision making," Information, vol. 11, no. 1, p. 5,2020 .
[15] P. Liu, M. Akram, and A. Sattar, "Extensions of prioritized weighted aggregation operators for decision-making under complex q-Rung orthopair fuzzy information," Journal of Intelligent and Fuzzy Systems, vol. 39, no. 5, pp. 7469-7493, 2020.

[16] N. Jan, K. Ullah, T. Mahmood et al., "Some root level modifications in interval valued fuzzy graphs and their generalizations including neutrosophic graphs," Mathematics, vol. 7, no. 1, p. 72, 2019.

[17] P. Liu, S. M. Chen, and P. Wang, "Multiple-attribute group decision-making based on q-Rung orthopair fuzzy power maclaurin symmetric mean operators," IEEE Transactions on Systems, Man, and Cybernetics: Systems, vol. 50, no. 10, pp. 3741-3756, 2018.

[18] P. Liu and P. Wang, "Multiple-attribute decision-making based on Archimedean Bonferroni operators of q-Rung orthopair fuzzy numbers," IEEE Transactions on Fuzzy Systems, vol. 27, no. 5, pp. 834-848, 2018.

[19] I. Yusuf, S. Umar, and K. Suleiman, "Analysis of structural failures," Building Bridges, vol. 3, no. 4, pp. 11-31, 2020.

[20] K. Ullah, T. Mahmood, and H. Garg, "Evaluation of the performance of search and rescue robots using T-spherical fuzzy Hamacher aggregation operators," International Journal of Fuzzy Systems, vol. 22, no. 2, pp. 570-582, 2020.

[21] P. Talukdar, S. Goala, P. Dutta, and B. Limboo, "Fuzzy multicriteria decision making in medical diagnosis using an advanced distance measure on linguistic Pythagorean fuzzy sets," Annals of Optimization Theory and Practice, vol. 3, no. 4, pp. 113-131, 2020.

[22] H. Garg, K. Ullah, T. Mahmood, N. Hassan, and N. Jan, "Tspherical fuzzy power aggregation operators and their applications in multi-attribute decision making," Journal of Ambient Intelligence and Humanized Computing, pp. 1-14, 2021.

[23] N. Jan, L. Zedam, T. Mahmood, and K. Ullah, "Cubic bipolar fuzzy graphs with applications," Journal of Intelligent \& Fuzzy Systems, vol. 37, no. 2, pp. 2289-2307, 2019.

[24] S. Zeng, Y. Hu, T. Balezentis, and D. Streimikiene, "A multicriteria sustainable supplier selection framework based on neutrosophic fuzzy data and entropy weighting," Sustainable Development, vol. 28, no. 5, pp. 1431-1440, 2020.

[25] A. Rosenfeld, "Fuzzy graphs," in Fuzzy Sets and their Applications to Cognitive and Decision Processes, pp. 77-95, Academic Press, Cambridge, MA, USA, 1975.

[26] R. Parvathi and M. G. Karunambigai, "Intuitionistic fuzzy graphs," in Computational Intelligence, Theory and Applications, pp. 139-150, Springer, Berlin, Germany, 2006.

[27] R. Parvathi, M. G. Karunambigai, and K. T. Atanassov, "Operations on intuitionistic fuzzy graphs," in Proceedings of the FUZZ-IEEE 2009, IEEE International Conference on Fuzzy Systems, pp. 1396-1401, Jeju Island, Korea, August 2009.

[28] M. Akram, A. Habib, F. Ilyas, and J. Dar, "Specific types of Pythagorean fuzzy graphs and application to decision-making," Mathematical and Computational Applications, vol. 23, no. 3, p. 42, 2018.

[29] A. Habib, M. Akram, and A. Farooq, "Q-rung orthopair fuzzy competition graphs with application in the soil ecosystem," Mathematics, vol. 7, no. 1, p. 91, 2019.

[30] A. Luqman, M. Akram, and A. N. Al-Kenani, "q-rung orthopair fuzzy hypergraphs with applications," Mathematics, vol. 7, no. 3, p. 260, 2019.

[31] P. Thirunavukarasu, R. Suresh, and K. K. Viswanathan, "Index," Insight into Fuzzy Modeling, vol. 10, no. 1, pp. 243-248, 2016. 
[32] N. Yaqoob, M. Gulistan, S. Kadry, and H. A. Wahab, "Complex intuitionistic fuzzy graphs with application in cellular network provider companies," Mathematics, vol. 7, no. 1, p. $35,2019$.

[33] M. Akram and S. Naz, "A novel decision-making approach under complex Pythagorean fuzzy environment," Mathematical and Computational Applications, vol. 24, no. 3, p. 73, 2019.

[34] M. Akram and A. Khan, "Complex Pythagorean Dombi fuzzy graphs for decision making," Granular Computing, vol. 6, pp. 645-669, 2021.

[35] M. Akram and A. Sattar, "Competition graphs under complex Pythagorean fuzzy information," Journal of Applied Mathematics and Computing, vol. 63, no. 1, pp. 543-583, 2020.

[36] S. Samanta and M. Pal, "Fuzzy planar graphs," IEEE Transactions on Fuzzy Systems, vol. 23, no. 6, pp. 1936-1942, 2015.

[37] T. Pramanik, S. Samanta, and M. Pal, "Interval-valued fuzzy planar graphs," International Journal of Machine Learning and Cybernetics, vol. 7, no. 4, pp. 653-664, 2016.

[38] S. Samanta, M. Pal, and A. Pal, "New concepts of fuzzy planar graph," International Journal of Advanced Research in Artificial Intelligence, vol. 3, no. 1, pp. 52-59, 2014.

[39] N. Alshehri and M. Akram, "Intuitionistic fuzzy planar graphs," Discrete Dynamics in Nature and Society, vol. 2014, Article ID 397823, 2014.

[40] M. Akram, J. Dar, and A. Farooq, "Planar graphs under Pythagorean fuzzy environment," Mathematics, vol. 6, no. 12, p. 278, 2018.

[41] M. Akram, A. Bashir, and S. Samanta, "Complex pythagorean fuzzy planar graphs," International Journal of Applied and Computational Mathematics, vol. 6, pp. 1-27, 2020.

[42] M. Akram, A. Sattar, and A. B. Saeid, "Competition graphs with complex intuitionistic fuzzy information," Granular Computing, pp. 1-23, 2021.

[43] T. Mahmood, K. Ullah, Q. Khan, and N. Jan, “An approach toward decision-making and medical diagnosis problems using the concept of spherical fuzzy sets," Neural Computing and Applications, vol. 31, no. 11, pp. 7041-7053, 2019.

[44] A. Guleria and R. K. Bajaj, "T-spherical fuzzy graphs: operations and applications in various selection processes," Arabian Journal for Science and Engineering, vol. 45, no. 3, pp. 2177-2193, 2020. 